\title{
Stationary solutions of driven fourth- and sixth-order Cahn-Hilliard type equations
}

\author{
$\begin{array}{lll}\text { M. D. Korzec* } & \text { P. L. Evans } & \text { A. Münch } \\ \ddagger & \text { B. Wagner } & \end{array}$
}

November 1, 2018

\begin{abstract}
New types of stationary solutions of a one-dimensional driven sixthorder Cahn-Hilliard type equation that arises as a model for epitaxially growing nano-structures such as quantum dots, are derived by an extension of the method of matched asymptotic expansions that retains exponentially small terms. This method yields analytical expressions for far-field behavior as well as the widths of the humps of these spatially non-monotone solutions in the limit of small driving force strength which is the deposition rate in case of epitaxial growth. These solutions extend the family of the monotone kink and antikink solutions. The hump spacing is related to solutions of the Lambert $W$ function.

Using phase space analysis for the corresponding fifth-order dynamical system, we use a numerical technique that enables the efficient and accurate tracking of the solution branches, where the asymptotic solutions are used as initial input.

Additionally, our approach is first demonstrated for the related but simpler driven fourth-order Cahn-Hilliard equation, also known as the convective Cahn-Hilliard equation.
\end{abstract}

Keywords: convective Cahn-Hilliard, quantum dots, exponential asymptotics, matching, dynamical systems

\section{Introduction}

A paradigm for phase separating systems such as binary alloys is the CahnHilliard equation for the phase fraction $u$

$$
u_{t}+\left(Q(u)+\varepsilon^{2} u_{x x}\right)_{x x}=0
$$

${ }^{*}$ Corresponding author, Weierstrass Institute for Applied Analysis and Stochastics (WIAS), D-10117 Berlin, Germany (korzec@wias-berlin.de).

${ }^{\dagger}$ Institute for Mathematics, Humboldt University of Berlin, D-10099 Berlin, Germany (pevans@mathematik.hu-berlin.de).

${ }^{\ddagger}$ School of Mathematical Sciences, University of Nottingham, UK (andreas.muench@nottingham.ac.uk).

$\S$ Weierstrass Institute for Applied Analysis and Stochastics (WIAS), D-10117 Berlin, Germany (wagnerb@wias-berlin.de). 
where $Q(u)$ is the derivative of the double-well potential $\mathcal{F}$, typically

$$
Q(u)=\mathcal{F}^{\prime}(u)=u-u^{3} .
$$

The long-time dynamics are characterized by the logarithmically slow coarsening process of phases, corresponding to local minima of the potential, separated by interfaces of width $\varepsilon$. This process is well described by the motion of equidistantly spaced smoothed shock solutions or kinks ("positive kinks") and antikinks ("negative kinks") which connect the local minimum of $\mathcal{F}(u)$ at $u=-1$ to that at $u=1$ and vice versa.

In recent years, an extension of this model has been studied for the case when the phase separating system is driven by an external field [16, 27]. In one space dimension it can be written as

$$
u_{t}-\nu u u_{x}+\left(Q(u)+\varepsilon^{2} u_{x x}\right)_{x x}=0,
$$

where $\nu$ denotes the strength of the external field. This equation, the convective Cahn-Hilliard (CCH) equation, also arises as a model for the evolution of the morphology of steps on crystal surfaces [21], and the growth of thermodynamically unstable crystal surfaces into a melt with kinetic undercooling and strongly anisotropic surface tension [9, 11, 17].

The dynamics of this model as $\nu \rightarrow 0$ are characterized by coarsening, as is typical for the Cahn-Hilliard equation $(\nu=0)$ [7, 26]. If $\nu \rightarrow \infty$ using the transformation $u \mapsto u / \nu$ in (1.3) one obtains the Kuramoto-Sivashinski equation, which is a well-known model for spatio-temporal chaotic dynamics (see e.g. 10] and references therein). Recently, Eden and Kalantarov [6] also established the existence of a finite-dimensional inertial manifold for the $\mathrm{CCH}$ equation, viewed as an infinite-dimensional dynamical system.

A related higher-order evolution equation arises in the context of epitaxially growing thin films (for a review on self-ordered nano-structures on crystal surfaces see Shchukin and Bimberg [24]). Here, the formation of quantum dots and their faceting has been described by the sixth-order equation

$$
u_{t}-\nu u u_{x}-\left(Q(u)+\varepsilon^{2} u_{x x}\right)_{x x x x}=0,
$$

where $u$ denotes the surface slope, $\nu$ is proportional to deposition rate 22] and $Q(u)$ is given with (1.2), it is assumed to have this form from now on throughout the paper. The high order derivatives are a result of the additional regularization energy which is required to form an edge between two plane surfaces with different orientations. This implies that the crystal surface tension also depends on curvature, which becomes very high at edges as the parameter $\varepsilon$ goes to zero. In analogy to the Cahn-Hilliard equation, here the phases are the orientations of the facets. This higher-order convective Cahn-Hilliard (HCCH) equation shares many properties with the $\mathrm{CCH}$ equation. In both cases the dynamics are described by conserved order parameters if $\nu=0$. They also share characteristic coarsening dynamics as $\nu \rightarrow 0$ and chaotic dynamics as $\nu$ gets large. To understand the complicated structure of the solutions it is 
instructive to study first the stationary solutions and their stability as it has been done for the $\mathrm{CCH}$ equation [16, 28]. For small $\nu$ the stationary solutions for both equations have been characterized by the monotone kink and antikink solutions [16, 22]. Recently new spatially non-monotone solutions were found for the lower order equation [28]. In this study we establish that the $\mathrm{HCCH}$ equation also possesses such non-monotone solutions. We show this by using phase-space methods for the corresponding fifth-order boundary value problem. We use the expression "simple" or "monotone" for a solution that connects the maximal value of $u(x)$ to the minimal value without any humps on the way down, although these extrema exist and lead to non-monotonicity even for simple (anti-)kink solutions of the $\mathrm{HCCH}$ equation.

Since the treatment of this high-order problem is not straightforward, one part of this study is concerned with the development of an approach that accurately locates the heteroclinic connections in the five-dimensional phase space. We find that these stationary solutions develop oscillations whose width and amplitude increase as $\nu \rightarrow 0$.

In the second part of this study we derive an analytic expression for the width and amplitude within the asymptotic regime of small external field strength. For the $\mathrm{CCH}$ equation we find that the width has a logarithmic dependency on the strength of the external field, while for the $\mathrm{HCCH}$ equation our analysis yields a dependency in terms of the Lambert $W$ function. In order to arrive at these expressions we solve the fifth-order equation by a combination of the method of matched asymptotic expansions and exponential asymptotics. We first demonstrate our approach for the third-order boundary value problem arising from the $\mathrm{CCH}$ equation. Our approach generalizes the work by Lange [15] to higherorder singularly perturbed nonlinear boundary-value problems, where standard application of matched asymptotics is not able to locate the position of interior layers that delimit the oscillations of the non-monotone solutions.

Reyna and Ward [19] previously developed an approach to resolve the internal layer structure of the solutions to the boundary-value problem for the related Cahn-Hilliard and viscous Cahn-Hilliard equations. The approach is based on a method due to Ward 25] who uses a "near" solvability condition for the corresponding linearized problem in his asymptotic analysis, and who was inspired by an earlier variational method [13] and work by O'Malley [18] and Rosenblat et al. 20], who investigated the problem of spurious solutions to singular perturbation problems of second-order nonlinear boundary-value problems [3]. Moreover, for the related Kuramoto-Sivashinsky equation, a multiple-scales analysis of the corresponding third-order nonlinear boundary-value problem by Adams et al. [1] shows that the derivation of monotone and oscillating travelingwave solutions involve exponentially small terms; their method is based on an analysis of the Stokes phenomenon of the corresponding problem in the complex plane (see Howls et al. [12] for an introduction).

In what follows we begin with the phase space analysis for the $\mathrm{CCH}$ equation in section 2 , followed by the asymptotic treatment for $\nu \ll 1$. The asymptotic ideas used for the $\mathrm{CCH}$ equation are then applied to the $\mathrm{HCCH}$ equation in section 3 . The solutions obtained there are useful to serve as initial input for the 
numerical investigations of the branches of non-monotone solutions in section 4 . In this part we develop our numerical approach and then use it to identify new stationary solutions of the $\mathrm{HCCH}$ equation, these agree with the asymptotic theory. Finally we shortly sum the results up together with concluding remarks in the last section 5 .

\section{Stationary solutions of the convective Cahn- Hilliard equation}

The high-order term in the $\mathrm{CCH}$ equation represents the regularization of the internal layers of the solutions. For most of our investigations we consider the problem in the scaling of the internal layers, or inner scaling, where the $x$-coordinate is stretched about the location $x=\bar{x}$ of a layer according to

$$
x^{*}=\frac{x-\bar{x}}{\varepsilon} .
$$

In this scaling the $\mathrm{CCH}$ equation becomes (after dropping the "*")

$$
\varepsilon^{2} u_{t}-\frac{\delta}{2}\left(u^{2}\right)_{x}+\left(Q(u)+u_{x x}\right)_{x x}=0,
$$

where $\delta=\varepsilon \nu$. The stationary problem obtained by setting $u_{t}$ to zero can be integrated once, requiring that the solutions approach the constants $\pm \sqrt{A}$ as $x \rightarrow \mp \infty$, where $A$ is a constant of integration. That is, we consider the boundary value problem

$$
\frac{\delta}{2}\left(u^{2}-A\right)=\left(Q(u)+u_{x x}\right)_{x}
$$

together with the far-field conditions

$$
\lim _{x \rightarrow \pm \infty} u=\mp \sqrt{A}
$$

and vanishing derivatives in the same spatial limit. We refer to solutions of this system as antikinks. Monotone antikinks are known analytically [16], while recently, non-monotone connections were computed numerically by Zaks et al. [28]. We now briefly discuss the numerical approach to obtain these solutions. Here we concentrate on the regime where $0<\delta \ll 1$ in order to compare with the asymptotic solutions derived later on. For a bifurcation analysis for larger $\delta$ we refer to [28].

\subsection{Numerical solutions}

For the numerical solutions we will work with a rescaled version, where we set $u=\sqrt{A} c$ so that the equilibrium points do not depend on $A$, and for $Q(u)$ given by (1.2), (2.3) and (2.4) become

$$
\left(1-c^{2}\right)=-\frac{2}{\delta \sqrt{A}}\left(c_{x x}+c-A c^{3}\right)_{x}, \quad \lim _{x \rightarrow \pm \infty} c=\mp 1 .
$$


For this problem we find it most convenient to present a shooting method which enables us to track solution branches in the $(A, \delta)$ parameter plane. We transform (2.5) to a first order system $U^{\prime}=F(U)$, where $F: \mathbb{R}^{3} \rightarrow \mathbb{R}^{3}$ is the function

$$
F_{1}(U)=U_{2}, \quad F_{2}(U)=U_{3}, \quad F_{3}(U)=\left(3 A\left(U_{1}\right)^{2}-1\right) U_{2}+\frac{\delta \sqrt{A}}{2}\left(\left(U_{1}\right)^{2}-1\right) .
$$

We work in a three dimensional phase space and denote either vectors or whole trajectories therein with capital $U$ 's. We use the same notation for two different objects, because it will be clear from the context what is meant. Subscripts indicate the components.

The characteristic polynomials at the equilibrium points $U^{ \pm}=( \pm 1,0,0)^{T}$ are

$$
\mathcal{P}^{ \pm}(\lambda)=\left|\frac{d F}{d U}\left(U^{ \pm}\right)-\lambda I\right|=\lambda^{3}+\lambda(1-3 A) \mp \delta \sqrt{A}
$$

The signs of the real parts of the roots determine the dimension of the stable and unstable manifolds $W^{u}\left(U^{+}\right), W^{s}\left(U^{-}\right), W^{s}\left(U^{+}\right), W^{u}\left(U^{-}\right)$of the equilibrium points. The latter two are two-dimensional and so the existence of a kink is generic, while this is not the case for the antikinks. The dimensions of $W^{u}\left(U^{+}\right)$ and $W^{s}\left(U^{-}\right)$are one, so that the heteroclinic connections from the positive to the negative equilibrium arise from a codimension two intersection. This means that with the two parameters $A$ and $\delta$ we can expect only separated solutions when the manifolds intersect, but due to the reversibility properties which are discussed below the codimension reduces to one and we can expect separated solutions for the free parameter $A$ and a fixed $\delta$, hence one or several whole branches in the $(A, \delta)$ parameter-plane. An example of a non-monotone connection is sketched in figure 1, where the trajectories wind themselves in the phase space with a solution that exhibits 15 humps.

Reversibility and computations It is instructive to note that the solution of (2.5) is translation invariant, $c(x) \rightarrow c(x+L)$, and forms a reversible dynamical system, hence the solutions are invariant with respect to the transformation $x \rightarrow-x, c \rightarrow-c$, as has also been noted by Zaks et al. 28.

Let us consider generally a $k$-dimensional phase space, since the following discussion will be also useful in section 4 where we analyze the $\mathrm{HCCH}$ equation with its higher order system. The linear transformation

$$
R: \mathbb{R}^{k} \rightarrow \mathbb{R}^{k}, \quad R\left(U_{j}\right)=(-1)^{j} U_{j}, j=1, \ldots, k
$$

fulfills $R^{2}=I d$ and $R F(U)=-F(R U)$ for $k=3$ and (2.6) and represents the reversibility in the phase space. It is an involution (or a reflection) and its set of fixed points is the symmetric section of the reversibility, these are zero at odd components, $U_{i}=0$ for odd $i$. A solution that crosses such a point necessarily symmetric under $R$, and for each point $U$ on the connection there exists a corresponding transformed point $R U$ somewhere on the branch. In 


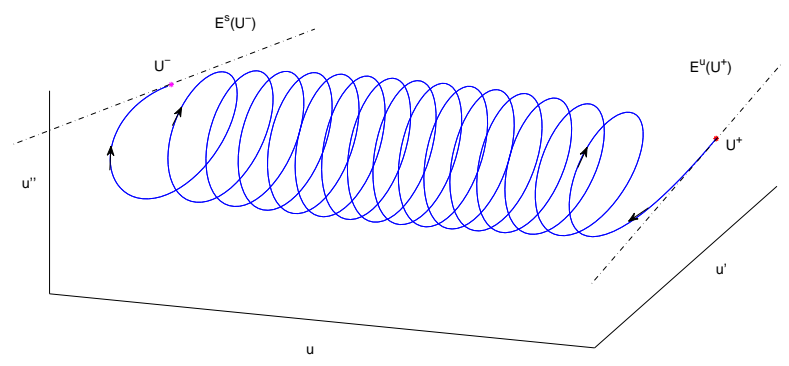

Figure 1: CCH: Antikink solutions connecting the hyperbolic equilibrium points $U^{+}$and $U^{-}$are sought in a 3 -D phase space. The unstable manifold emerging from $U^{+}, W^{u}\left(U^{+}\right)$is one-dimensional, as is the stable manifold $W^{s}\left(U^{-}\right)$. The approximating linearized spaces $E^{u}\left(U^{+}\right)$and $E^{s}\left(U^{-}\right)$are drawn as dash-dotted lines and are used in the computations.

fact there is an equivalence here since odd solutions necessarily cross a point in the symmetric section. It holds that $c$ and its even derivatives have to vanish in the point of symmetry $L$ because of the fulfilled equations $\frac{d^{2 m}}{d x^{2 m}} c(x+L)=$ $-\frac{d^{2 m}}{d x^{2 m}} c(-x+L), m=0,1, \ldots,\lfloor k / 2\rfloor$, and continuity of the solution and its derivatives.

From the above we conclude that with a shooting method we can stop integrating when we find a point with zero odd components, since the second half of the solution is then given by the set of transformed points under $R$. Hence we define the following distance function for a trajectory $U$ over the interval of integration which helps to find these points

$$
d_{A}(U)=\min _{x} \sqrt{\sum_{i \text { odd }} U_{i}(x)^{2}} .
$$

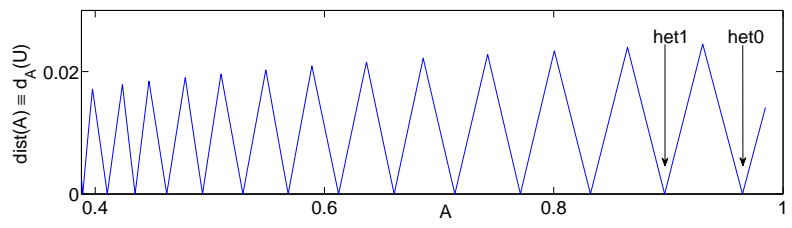

Figure 2: Distance function $d_{A}$ defined by (2.9) depending on $A$ with fixed $\delta=0.05$, showing the first 14 zeros corresponding to het 0 to het $t_{13}$.

The minimization of $d_{A}(U)$ over the free parameter, $\min _{A} d_{A}(U)$, must result in the value zero for an anti-symmetric heteroclinic solution. We can use this 

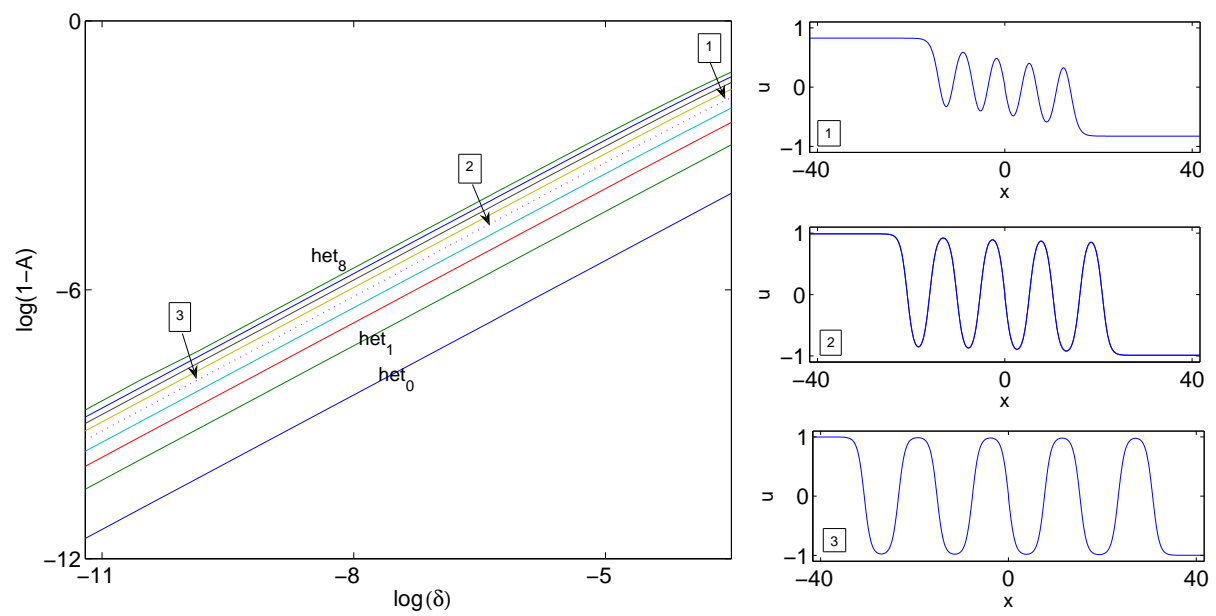

Figure 3: Parameter plane, $\log (\delta)$ for the $\mathrm{x}-$ and $\log (1-A)$ for the $\mathrm{y}$-axis, for the $\mathrm{CCH}$ equation for the first 9 antikink solutions $h_{e} t_{k}, k=0,1 \ldots, 8$. The graphs on the right show the shapes of representative het 4 solutions, hence those on the fifth line from below, for the approximate $(A, \delta)$ tuples $(0.8259,0.0289)$, $(0.9893,0.0017)$ and $\left(0.9998,2.6457 \cdot 10^{-5}\right)$.

condition for shooting and BVP formulations, for both the $\mathrm{CCH}$ and later the $\mathrm{HCCH}$ equation in section 4.

For a fixed value of $\delta$ and a range of different $A$ we follow the relevant branch of $W^{u}\left(U^{+}\right)$by shooting from an initial point $U^{+} \pm \epsilon v$ near $U^{+}$, where $v$ is a unit eigenvector corresponding to the positive eigenvalue of $d F / d U_{\mid U=U^{+}}$and $\epsilon \ll 1$. We stop the integration if a certain threshold value for $\left|U_{1}\right|$ is crossed. Figure 2 shows $d_{A}(U)$ as a function of $A$ for $\delta=0.05$.

At this point we have heteroclinic connections for one fixed value of $\delta$ which we denote by $h^{2} t_{k}, k=0,1, \ldots$ (using the notation in Zaks et al. [28]). het is the analytical, monotone tanh solution while het ${ }_{k}$ has $k$ humps on the way down from $\sqrt{A}$ to $-\sqrt{A}$. We will use the same terminology for the solution structure of the stationary $\mathrm{HCCH}$ problem in section 4. Here, a het $k_{k}$ solution corresponds to the $k$ th zero from the right in figure 2 We then follow the roots of the distance function by linearly extrapolating to a new guess for $A$ and use a bisection algorithm to converge fast to the next root. Figure 3 shows a portion of the $(A, \delta)$ parameter-plane, where we concentrate on very small values of $\delta$, or differently interpreted, on the bifurcation of the various spiraling $\mathrm{CCH}$ orbits from the heteroclinic connections of the $\mathrm{CH}$ equation in its one dimension smaller phase space. 


\section{$2.2 \quad$ Asymptotic internal layer analysis}

For the asymptotic analysis we use a slightly different scaling than for the numerical treatment. Here, we let

$$
x^{*}=\frac{x-\bar{x}}{\sqrt{2} \varepsilon}
$$

denote the inner variable about a layer located at $x=\bar{x}$. For the stationary problem we then obtain

$$
\left(u^{\prime \prime}+2 Q(u)\right)^{\prime}=\delta \sqrt{2}\left(u^{2}-A\right)
$$

instead of (2.3), where ${ }^{\prime}=d / d x^{*}$. For later comparisons of the numerical and asymptotic results we have to keep in mind that the spatial scales differ by a factor of $\sqrt{2}$.

We point out that the problem considered here shares the internal layer structure of the singular perturbation problems discussed by Lange [15], and we will make use and extend this ansatz for our situation. This will also prove useful to understand the approach taken for the $\mathrm{HCCH}$ problem in section 3.1 since there we have to carefully combine the exponential matching with the conventional matching procedure when matching the two regions. For both problems the asymptotic analysis can be conveniently carried out in terms of the small parameter $\delta$.

In the following analysis we consider the simplest case of a non-monotone solution with only one hump, as illustrated in figure 4 we note that non-monotone solutions with more oscillations can be treated similarly.

\subsubsection{The 1-hump solution}

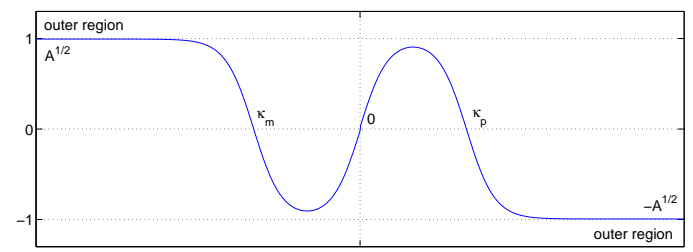

Figure 4: Sketch of a 1-hump, or het ${ }_{1}$ solution showing the general setup for the matching procedure for the $\mathrm{CCH}$ and $\mathrm{HCCH}$ equations.

We observe that the 1-hump solution has three internal layers, one at $\kappa_{m}<0$, one at $\kappa_{p}$ and one at the symmetry point in between. Since the solution is point symmetric we can choose this point to be $x=0$ and it will be enough to only discuss the two layers at $\kappa_{m}$ and zero and then match them to the outer solution. 
Internal layer near $\kappa_{m}$ For the first internal layer at $\kappa_{m}$ we let

$$
x_{m}=\frac{x}{\sqrt{2} \varepsilon}-\frac{\bar{\kappa}_{m}}{\sqrt{2}},
$$

where $\bar{\kappa}_{m}<0$ and set

$$
\kappa_{m}=\bar{\kappa}_{m}+\sqrt{2} \sum_{k=1}^{\infty} \delta^{k} \kappa_{m k}
$$

so that to leading order the location where the solution crosses zero is $\bar{\kappa}_{m}$ and the additional terms account for the corrections due to the higher order problems.

With $u_{m}\left(x_{m}\right)=u\left(\varepsilon\left(\bar{\kappa}_{m}+\sqrt{2} x_{m}\right)\right)$ the governing equation becomes

$$
u_{m}^{\prime \prime \prime}+2 Q^{\prime}\left(u_{m}\right)=\delta \sqrt{2}\left(u_{m}^{2}-A\right), \quad \text { where } \quad \prime=\frac{d}{d x_{m}} .
$$

For the boundary condition where $u_{m}$ crosses zero we have

$$
u_{m}\left(\frac{\kappa_{m}-\bar{\kappa}_{m}}{\sqrt{2}}\right)=0
$$

and the condition towards $-\infty$ is

$$
\lim _{x_{m} \rightarrow-\infty} u_{m}\left(x_{m}\right)=\sqrt{A} .
$$

We now assume $u_{m}\left(x_{m}\right)$ can be written as the following asymptotic expansion, valid near $\kappa_{m}$

$$
u_{\alpha}\left(x_{\alpha}\right)=u_{\alpha 0}\left(x_{\alpha}\right)+\sum_{k=1}^{\infty} \delta^{k} u_{\alpha k}\left(x_{\alpha}\right)
$$

with $\alpha=m$ here. Additionally, we assume $A$ has the asymptotic expansion

$$
A=1+\delta A_{1}+O\left(\delta^{2}\right) .
$$

Observe that from (2.16) and (2.18)

$$
\lim _{x_{m} \rightarrow-\infty} u_{m}\left(x_{m}\right)=\lim _{x_{m} \rightarrow-\infty} u_{m 0}\left(x_{m}\right)+\sum_{k=1}^{\infty} \delta^{k} u_{m k}\left(x_{m}\right)=1+\frac{1}{2} \sum_{k=1}^{\infty} \delta^{k} A_{k} .
$$

To leading order in $\delta$ we get the problem for the Cahn-Hilliard equation

$$
\begin{aligned}
& u_{m 0}^{\prime \prime \prime}+2 Q^{\prime}\left(u_{m 0}\right)=0 \\
& u_{m 0}(0)=0 \quad \text { and } \quad \lim _{x_{m} \rightarrow-\infty} u_{m 0}\left(x_{m}\right)=1
\end{aligned}
$$


with the unique solution $u_{m 0}\left(x_{m}\right)=-\tanh \left(x_{m}\right)$. Next, the problem of order $\delta$ is

$$
\begin{aligned}
& \left(\mathcal{L}\left(u_{m 1}, x_{m}\right)\right)^{\prime}=\sqrt{2}\left(\tanh ^{2}\left(x_{m}\right)-1\right) \\
& u_{m 1}(0)=\kappa_{m 1} \text { and } \lim _{x_{m} \rightarrow-\infty} u_{m 1}\left(x_{m}\right)=\frac{A_{1}}{2},
\end{aligned}
$$

where $\kappa_{m 1}$ and $A_{1}$ are constants to be exponentially matched and the operator $\mathcal{L}$ is defined by

$$
\mathcal{L}(v, z)=v^{\prime \prime}+2\left(1-3 \tanh ^{2}(z)\right) v,
$$

and $z=x_{m}, v=u_{m 1}$ and $\iota=d / d x_{m}$. Note that the first boundary condition is obtained by expanding (2.15)

$$
\begin{aligned}
u_{m}\left(\sum_{k=1}^{\infty} \delta^{k} \kappa_{m k}\right) & =u_{m}\left(\delta \kappa_{m 1}+\delta^{2} \kappa_{m 2}+O\left(\delta^{3}\right)\right) \\
& =u_{m 0}(0)+\delta\left(\kappa_{m 1} u_{m 0}^{\prime}(0)+u_{m 1}(0)\right)+O\left(\delta^{2}\right)
\end{aligned}
$$

so that collecting the terms of order $\delta$ gives

$$
u_{m 1}(0)=-\kappa_{m 1} u_{m 0}^{\prime}(0)=\kappa_{m 1} .
$$

Next, we integrate (2.21) once to obtain

$$
\mathcal{L}\left(u_{m 1}, x_{m}\right)=f_{m}\left(x_{m}\right),
$$

where $f_{m}\left(x_{m}\right)=-\sqrt{2} \tanh \left(x_{m}\right)+c_{m}$. Taking the limit of this equation to $-\infty$ yields $c_{m}=-\sqrt{2}-2 A_{1}$ so that

$$
f_{m}\left(x_{m}\right)=-\sqrt{2}\left(\tanh \left(x_{m}\right)+1\right)-2 A_{1} .
$$

The homogeneous solutions of (2.24) are

$$
\begin{aligned}
& \phi_{m}\left(x_{m}\right)=-u_{m 0}^{\prime}\left(x_{m}\right)=1-\tanh ^{2}\left(x_{m}\right), \\
& \psi_{m}\left(x_{m}\right)=\left(\int_{0}^{x_{m}} \frac{d z}{\phi_{m}^{2}(z)}\right) \phi_{m}\left(x_{m}\right) .
\end{aligned}
$$

Also note that $\lim _{x_{m} \rightarrow-\infty} \phi_{m}\left(x_{m}\right)=0$ and $\psi_{m}(0)=0$. At this stage it is convenient to choose the inhomogeneous solution that remains bounded as $x_{m} \rightarrow-\infty$ and vanishes at $x_{m}=0$ which is satisfied by

$$
\varphi_{\alpha}\left(x_{\alpha}\right)=\psi_{\alpha}\left(x_{\alpha}\right) \int_{-\infty}^{x_{\alpha}} \phi_{\alpha} f_{\alpha} d z-\phi_{\alpha}\left(x_{\alpha}\right) \int_{0}^{x_{\alpha}} \psi_{\alpha} f_{\alpha} d z
$$

with $\alpha=m$. Hence, the unique solution for (2.21) is the linear combination

$$
u_{m 1}\left(x_{m}\right)=-\kappa_{m 1} \phi_{m}\left(x_{m}\right)+\varphi_{m}\left(x_{m}\right) .
$$


Internal layer near $x=0 \quad$ For the internal layer near the origin we proceed as above. Here, we stretch the independent variable as

$$
x_{0}=\frac{x}{\sqrt{2} \varepsilon}
$$

and construct an asymptotic expansion (2.17) near $x=0$ with $\alpha=0$ for the solution of the problem

$$
u_{0}^{\prime \prime \prime}+2 Q^{\prime}\left(u_{0}\right)=\delta \sqrt{2}\left(u_{0}^{2}-A\right), \quad \text { where } \quad \prime=\frac{d}{d x_{0}} .
$$

We note that the point $x=0$ is assumed to be the symmetry point of the complete solution, hence here we require

$$
u_{0}(0)=0 \quad \text { and } \quad u_{0}^{\prime \prime}(0)=0 .
$$

In anticipation of the exponential matching we also require that $\lim _{x_{0} \rightarrow-\infty} u_{00}\left(x_{0}\right)=$ -1 , so that the solution to the leading order problem is $u_{00}\left(x_{0}\right)=\tanh \left(x_{0}\right)$ For the solution to $O(\delta)$ we find

$$
u_{01}\left(x_{0}\right)=b_{0} \psi_{0}\left(x_{0}\right)+\varphi_{0}\left(x_{0}\right)
$$

where $b_{0}$ is a further constant to be exponentially matched. Here, the homogeneous solutions are

$$
\phi_{0}\left(x_{0}\right)=-u_{00}^{\prime}\left(x_{0}\right) \quad \text { and } \quad \psi_{0}\left(x_{0}\right)=\left(\int_{0}^{x_{0}} \frac{d z}{\phi_{0}^{2}(z)}\right) \phi_{0}\left(x_{0}\right)
$$

and the inhomogeneous solution is defined by (2.28), where $\alpha=0$ and $f_{0}\left(x_{0}\right)=$ $-\sqrt{2} \tanh \left(x_{0}\right)$. They are chosen such that $\varphi_{0}(0)=0$ and $\varphi_{0}^{\prime \prime}(0)=0$, in fact we have $\lim _{x_{0} \rightarrow \pm \infty} \varphi_{0}\left(x_{0}\right)= \pm \sqrt{2} / 4$.

\subsubsection{Exponential matching}

Exponential matching requires that all exponentially small and exponentially growing terms have to be accounted for and matched. This means first that we have to express the variable $x_{0}$ in terms of $x_{m}$ (or vice versa). From the definitions of these variables it follows that

$$
x_{0}=x_{m}+\frac{\bar{\kappa}_{m}}{\sqrt{2}} .
$$

In particular, exponential terms in the solution $u_{0}\left(x_{0}\right)$ transform as $e^{2 x_{0}}=$ $e^{\sqrt{2} \bar{\kappa}_{m}} e^{2 x_{m}}$ and so forth for higher order exponential terms $e^{2 n x_{0}}$ or terms with different signs in the exponent.

Now note that as $x_{0} \rightarrow-\infty$ the leading and $O(\delta)$ solutions can be written as

$$
u_{00}\left(x_{0}\right)=-1+2 e^{2 x_{0}}-O\left(e^{4 x_{0}}\right)
$$


and with $\bar{\mu}=\left(\frac{3}{2} b_{0}+\sqrt{2}\right)$

$$
u_{01}\left(x_{0}\right)=-\frac{1}{4} \bar{\mu}-\frac{b_{0}}{16} e^{-2 x_{0}}+\left(\frac{13}{16} b_{0}+\frac{1}{\sqrt{2}}+\bar{\mu} x_{0}\right) e^{2 x_{0}}+O\left(e^{4 x_{0}}\right) .
$$

Written in $x_{m}$ variables the solution

$$
\begin{aligned}
u_{0}\left(x_{m}\right)= & -1+2 e^{2 x_{m}} e^{\sqrt{2} \bar{\kappa}_{m}}+O\left(e^{2 \sqrt{2} \bar{\kappa}_{m}}\right) \\
+ & \delta\left(-\frac{1}{4} \bar{\mu}-\frac{b_{0}}{16} e^{-2 x_{m}} e^{-\sqrt{2} \bar{\kappa}_{m}}+\left(\frac{13}{16} b_{0}+\frac{1}{\sqrt{2}}+\bar{\mu}\left(x_{m}+\frac{\bar{\kappa}_{m}}{\sqrt{2} \varepsilon}\right)\right) e^{2 x_{m}} e^{\sqrt{2} \bar{\kappa}_{m}}\right. \\
& \left.+O\left(e^{2 \sqrt{2} \bar{\kappa}_{m}}\right)\right)+O\left(\delta^{2}\right)
\end{aligned}
$$

has to be exponentially matched to

$$
\begin{aligned}
u_{m}\left(x_{m}\right)= & -1+2 e^{-2 x_{m}}+O\left(e^{-4 x_{m}}\right) \\
& +\delta\left(-\left(A_{1}+\frac{\sqrt{2}}{4}\right)-\frac{1}{4}\left(A_{1}+\frac{1}{\sqrt{2}}\right) e^{2 x_{m}}+\left(\frac{7}{2} A_{1}+\frac{5}{4} \sqrt{2}+4 \kappa_{m 1}\right) e^{-2 x_{m}}\right. \\
& \left.-\left(3 A_{1}+\frac{1}{\sqrt{2}}\right) x_{m} e^{-2 x_{m}}+O\left(e^{-4 x_{m}}\right)\right)+O\left(\delta^{2}\right)
\end{aligned}
$$

as $x_{m} \rightarrow \infty$. While we have already anticipated matching of the constants during the derivation of the leading order solutions, the constant terms of the $O(\delta)$ solutions are first to be matched. Matching to the exponential terms in (2.39) entails a rearranging of terms of different orders of magnitude in the expansion (2.38). In particular, the first exponential term to leading order in (2.39) matches the second term of $O(\delta)$ in (2.38), the second and largest exponential term of $O(\delta)$ in (2.39) matches the second term of the leading order in (2.38), and so forth. Summarizing, we obtain

$$
\frac{1}{4}\left(\frac{3}{2} b_{0}+\sqrt{2}\right)=A_{1}+\frac{\sqrt{2}}{4}, \quad-\rho \frac{b_{0}}{16}=2, \quad-\frac{\rho}{4}\left(A_{1}+\frac{1}{\sqrt{2}}\right)=2,
$$

where we denote $\rho=\delta e^{-\sqrt{2} \bar{\kappa}_{m}}$. Solving yields

$$
\rho=4 \sqrt{2}, \quad A_{1}=-\frac{3}{\sqrt{2}} \quad \text { and } \quad b_{0}=-\frac{8}{\sqrt{2}} .
$$

We observe that we have determined the $O(\delta)$ correction $A_{1}$. Additionally, we now know that $\delta e^{-\sqrt{2} \bar{\kappa}_{m}}=4 \sqrt{2}$, hence

$$
\bar{\kappa}_{m}=\frac{\ln (\delta)}{\sqrt{2}}-\frac{\ln (4 \sqrt{2})}{\sqrt{2}}
$$

and if we recall (2.13) and $\kappa_{m}<0$ then the width of the hump is $-\kappa_{m}$, where

$$
\kappa_{m}=\frac{\ln (\delta)}{\sqrt{2}}-\frac{\ln (4 \sqrt{2})}{\sqrt{2}}+O(\delta)
$$




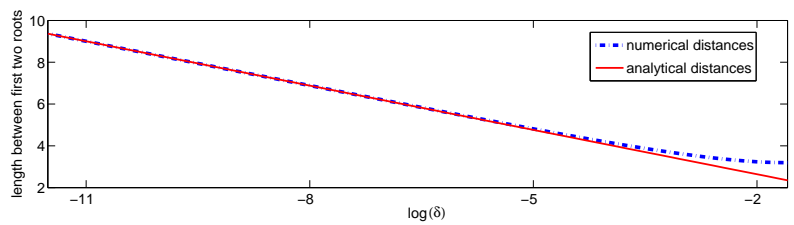

Figure 5: Distances between the first two roots of the het ${ }_{1}$ solutions versus $\log (\delta)$ together with the width predicted by the asymptotic formula (2.43).

Further constants, such as $\kappa_{m 1}$ are found by including higher exponential terms and expansions of the higher order problems. Finally we note that making use of the symmetry of the solution about the point $x=0$, the exponential matching of the solution near zero to the one near $\kappa_{p}$ proceeds analogously.

\subsubsection{Comparison of numerical and asymptotic solution}

For the comparison with the asymptotic solution we are interested mainly in the $h_{e} t_{1}$ solution which we derived in section 2.2.1. By numerical continuation of the shooting method, one obtains $N$ tuples $\left(A^{(j)}, \delta^{(j)}\right), j=1, \ldots, N$ in the param-

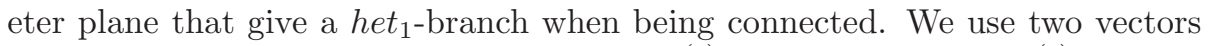
of parameters which we abbreviate $\mathbf{A}=\left(A^{(j)}\right)_{j=1, \ldots, N}$ and $\boldsymbol{\delta}=\left(\delta^{(j)}\right)_{j=1, \ldots, N}$ to confirm the formulas we obtained in the previous section. Further we make use of a distance vector $\mathbf{K}=\left(K^{(j)}\right)_{j=1, \ldots, N}$. $\mathbf{K}$ contains the distances between the zero crossings of the solutions, or in context of the asymptotics section (see figure (4) $K^{(j)} \approx\left|\kappa_{m}\left(\delta^{(j)}\right)\right|$. To obtain the relation between $A$ and $\delta$ and the evolution of the distances we solve the least squares problems

$$
\min _{\mu_{1}}\left\|\left(\mathbf{1}-\mu_{1} \boldsymbol{\delta}\right)-\mathbf{A}\right\|_{2}^{2} \quad \text { and } \quad \min _{\eta_{1}, \eta_{2}}\left\|\eta_{1} \log \left(\boldsymbol{\delta} \eta_{2}\right)-\mathbf{K}\right\|_{2}^{2},
$$

hence we assume a linear law for the $A$-values in $\delta$ and a general logarithmic law for the distances. We obtain

$$
A \approx 1-2.12 \delta \approx 1-\frac{3}{\sqrt{2}} \delta \quad \text { and } \quad K \approx-0.71 \log (0.18 \delta)
$$

which confirms the results from the analysis (2.41) and (2.43). We see the good match in the distance plot in figure 5

These results motivated us to obtain a general rule for the relation between the two parameters of the $\mathrm{CCH}$ equation for different stationary solutions. The numerically computed branches in figure 3 show that the slopes of the het $k_{k}$ branches are one when plotting $\log (\delta)$ against $\log (1-A)$, so that the relation $\log (\delta)+$ const $=\log (1-A)$ shows the linear dependence $A(k)=1+A_{1}(k) \delta$, where $A(k)$ is the $A$ value for the het ${ }_{k}$ solution and $A_{1}(k)$ its linear coefficient. We see that the dependence of $A_{1}$ with respect to the order of the heteroclinic connection $k$ behaves linearly and we obtain a general expression for the squared 
far field value of non-monotone het $_{k}$ solutions, it is given with

$$
A_{1}(k)=-\frac{2 k+1}{\sqrt{2}}
$$

\section{Matched and exponential asymptotics for the stationary $\mathrm{HCCH}$ equation}

As for the $\mathrm{CCH}$ equation we will perform our analysis of the internal layers in the inner scaling (2.10). From the stationary form of (1.4) we obtain the equation

$$
\left(u^{\prime \prime}+2 Q(u)\right)^{\prime \prime \prime}=-\delta 2^{3 / 2}\left(u^{2}-A\right),
$$

after integrating once and requiring that for an antikink $\lim _{x \rightarrow \pm \infty} u=\mp \sqrt{A}$ and setting $\delta=\epsilon^{3} \nu$ here. We consider the het (one hump) solution, and again make use of the point symmetry of the problem. Now however, unlike for the $\mathrm{CCH}$ equation, the solutions in the outer region are not just constants. Here, we have to introduce an outer layer to the left of the inner layer about $\kappa_{m}$, see also figure 4 for the case of a 1-hump solution. In the following subsections we first briefly derive the solution to this outer problem and match it to the solution to the inner problem near $\kappa_{m}$. The remaining degrees of freedom are then used to exponentially match it to a second inner layer near $x=0$.

It has been demonstrated in 22] for monotone antikink solutions of the $\mathrm{HCCH}$ equation, that it is necessary to match terms up to order $\delta$ in order to obtain the correction $A_{1}$, given the asymptotic expansion of $A$

$$
A=1+\sum_{k=1}^{\infty} \delta^{k / 3} A_{k} .
$$

Here, for the non-monotone antikinks we have to match inner and outer solutions and then also exponentially match the inner layers. This has to be carried through iteratively up to three orders of magnitude in order to obtain not only the correction $A_{1}$ but also the expression for the width of the humps.

\subsection{The 1-hump solution for the $\mathrm{HCCH}$ equation}

We start by shifting to the inner coordinates that describe the region near $\kappa_{m}$, which is to be matched to the outer region. Again defining $x_{m}$ by 2.12), the governing equation in this inner region is

$$
\left(u_{m}^{\prime \prime}+2 Q\left(u_{m}\right)\right)^{\prime \prime \prime}=-2^{3 / 2} \delta\left(u_{m}^{2}-A\right) \quad \text { where } \quad,=\frac{d}{d x_{m}} .
$$

For the boundary conditions we again place $\kappa_{m}$ near the point where $u_{m}$ crosses zero, i.e.

$$
u_{m}\left(\frac{\kappa_{m}-\bar{\kappa}_{m}}{\sqrt{2}}\right)=0
$$


The condition towards $-\infty$ is not as trivial as for the $\mathrm{CCH}$ equation but needs to be matched to the outer solution in the region to the left of $\kappa_{m}$ (or to the right of $\kappa_{p}$, taking account of symmetry).

For the outer region (see figure 4), where $x_{m}$ becomes very large, we use the ansatz

$$
\xi=\delta^{1 / 3} x_{m} \quad \text { and } \quad Y(\xi ; \delta)=u_{m}\left(x_{m} ; \delta\right)
$$

and obtain the outer problem

$$
\left(\delta^{2 / 3} Y_{\xi \xi}+2 Q(Y)\right)_{\xi \xi \xi}=-2^{3 / 2}\left(Y^{2}-A\right)
$$

with the far field condition

$$
\lim _{\xi \rightarrow-\infty} Y(\xi)=\sqrt{A}
$$

The region near $x=0$, for which we use the variable $x_{0}$ from (2.30), is described by the problem

$$
\left(u_{0}^{\prime \prime}+2 Q\left(u_{0}\right)\right)^{\prime \prime \prime}=-2^{3 / 2} \delta\left(u_{0}^{2}-A\right) \quad \text { where } \quad \prime=\frac{d}{d x_{0}}
$$

The point $x=0$ is the point of symmetry of the solution. Here we require

$$
u_{0}(0)=0, \quad u_{0}^{\prime \prime}(0)=0 \quad \text { and } \quad u_{0}^{\prime \prime \prime \prime}(0)=0,
$$

plus additional conditions from the exponential matching to the internal layer near $\kappa_{m}$ as $x_{0} \rightarrow-\infty$, as we have shown for the $\mathrm{CCH}$ equation.

Here we assume the solutions to these three problems for $Y, u_{m}$ and $u_{0}$ can be represented by asymptotic expansions

$$
u_{\alpha}\left(x_{\alpha} ; \varepsilon\right)=u_{\alpha 0}\left(x_{\alpha}\right)+\sum_{k=1}^{\infty} \delta^{k / 3} u_{\alpha k}\left(x_{\alpha}\right), \quad \text { where } \quad \alpha=0, m
$$

valid near $\kappa_{m}$ and $x=0$, respectively, and

$$
Y(\xi ; \delta)=Y_{0}(\xi)+\sum_{k=1}^{\infty} \delta^{k / 3} Y_{k}(\xi)
$$

valid in the outer region, where we let

$$
\kappa_{m}=\bar{\kappa}_{m}+\sqrt{2} \sum_{k=1}^{\infty} \delta^{k / 3} \kappa_{m k} .
$$

To obtain solutions to the outer problem is straightforward [22], but in order to be more comprehensible we include the results in appendix A. The solutions to the other regions are discussed now. 


\subsubsection{Leading order}

To leading order in $\delta$ we get the problem

$$
\begin{aligned}
& \left(u_{m 0}^{\prime \prime}+2 Q\left(u_{m 0}\right)\right)^{\prime \prime \prime}=0 \\
& u_{m 0}(0)=0
\end{aligned}
$$

Matching to the leading order outer solution (A.2) $Y_{0}=1$ we find

$$
u_{m 0}\left(x_{m}\right)=-\tanh \left(x_{m}\right) .
$$

Its representation towards the internal layer about $x=0$ is given by

$$
u_{m 0}=-1+2 e^{-2 x_{m}}-2 e^{-4 x_{m}}+O\left(e^{-6 x_{m}}\right)
$$

as $x_{m} \rightarrow \infty$. The leading order problem for this region is

$$
\begin{aligned}
& \left(u_{00}^{\prime \prime}+2 Q\left(u_{00}\right)\right)^{\prime \prime \prime}=0 \\
& u_{00}(0)=0, \quad u_{00}^{\prime \prime}(0)=0 \quad \text { and } \quad u_{00}^{\prime \prime \prime \prime}(0)=0
\end{aligned}
$$

and its solution is

$$
u_{00}\left(x_{0}\right)=\tanh \left(x_{0}\right) .
$$

As $x_{0} \rightarrow-\infty$ its behavior is given by

$$
u_{00}=-1+2 e^{2 x_{0}}-2 e^{4 x_{0}}+O\left(e^{6 x_{0}}\right) .
$$

\section{$3.1 .2 \quad \mathrm{O}\left(\delta^{1 / 3}\right)$}

Internal layer near $\mathbf{x}=\boldsymbol{\kappa}_{\mathrm{m}}$ The expansion of (3.3) and (3.4) to order $\delta^{1 / 3}$ yields

$$
\begin{aligned}
& \mathcal{L}\left(u_{m 1}, x_{m}\right)=f_{m 1}\left(x_{m}\right) \\
& u_{m 1}(0)=-u_{m 0}^{\prime}(0) \kappa_{m 1}=\kappa_{m 1}
\end{aligned}
$$

where $\mathcal{L}$ is defined by (2.22) as for the $\mathrm{CCH}$ equation and

$$
f_{m 1}\left(x_{m}\right):=c_{1 m} x_{m}^{2}+c_{2 m} x_{m}+c_{3 m} .
$$

The homogenous solutions are therefore (2.26) and (2.27). The constants $c_{1 m}, c_{2 m}, c_{3 m}$ are obtained by three successive integrations of the ODE for $u_{m 1}$ obtained at this order. We choose the inhomogeneous solution so that it grows only algebraically as $x_{m} \rightarrow-\infty$ and vanishes at $x_{m}=0$. Particular solutions to (3.19b) are of the form

$$
\varphi_{\alpha j}\left(x_{\alpha}\right)=\psi_{\alpha}\left(x_{\alpha}\right) \int_{0}^{x_{\alpha}} \phi_{\alpha} f_{\alpha j} d z-\phi_{\alpha}\left(x_{\alpha}\right) \int_{0}^{x_{\alpha}} \psi_{\alpha} f_{\alpha j} d z+\gamma_{\alpha j} \psi_{\alpha}\left(x_{\alpha}\right),
$$

so that now we obtain $\varphi_{m 1}$ for $\alpha=m, j=1$ in (3.21) and

$$
\gamma_{m 1}=-\frac{\pi^{2}}{12} c_{1 m}+\ln (2) c_{2 m}-c_{3 m}
$$


Hence the solution is

$$
u_{m 1}\left(x_{m}\right)=-\kappa_{m 1} \phi_{m}\left(x_{m}\right)+\varphi_{m 1}\left(x_{m}\right) .
$$

We evaluate $\psi_{\alpha}, \phi_{\alpha}$ etc. and subsequent functions with the assistance of Maple. As $x_{m} \rightarrow-\infty$ the limiting behavior of $u_{m 1}$ is

$$
\begin{aligned}
u_{m 1}\left(x_{m}\right)= & -\frac{1}{8}\left(c_{1 m}+2 c_{3 m}\right)-\frac{1}{4} c_{2 m} x_{m}-\frac{1}{4} c_{1 m} x_{m}^{2} \\
+ & \left(\frac{1}{64}\left(-7 c_{1 m}-8 c_{3 m}+256 \kappa_{m 1}+30 c_{2 m}+4 c_{2 m} \pi^{2}-72 c_{1 m} \zeta(3)\right)\right. \\
& \left.+\frac{1}{16}\left(-6 c_{2 m}+15 c_{1 m}+24 c_{3 m}\right) x_{m}+\frac{1}{8}\left(6 c_{2 m}-3 c_{1 m}\right) x_{m}^{2}+\frac{1}{2} c_{1 m} x_{m}^{3}\right) e^{2 x_{m}} \\
& +O\left(e^{4 x_{m}}\right)
\end{aligned}
$$

where $\zeta$ is the Riemann Zeta function, and $u_{m 1}$ must match the outer solution which is given in the appendix by (A.10) and has only constant terms to this order. Hence we require $c_{2 m}=0$ and $c_{1 m}=0$. The matched solution is now

$$
\begin{aligned}
u_{m 1}^{(m)}\left(x_{m}\right) & =\left(1-\tanh ^{2}\left(x_{m}\right)\right) \kappa_{m 1} \\
& -\frac{c_{3 m}}{16}\left(-2 e^{6 x_{m}}+4+10 e^{2 x_{m}}-12 e^{4 x_{m}}-24 x_{m} e^{2 x_{m}}\right) \frac{e^{-2 x_{m}}}{\left(e^{2 x_{m}}+1\right)^{2}},
\end{aligned}
$$

where we denote by $u_{m 1}^{(m)}$ the solution that is obtained by matching to the outer solution $Y$. As we will see later, exponential matching to the inner solution $u_{0}$, i.e. as $x_{m} \rightarrow \infty$, where we find

$$
\begin{aligned}
u_{m 1}^{(m)}\left(x_{m}\right)= & \frac{1}{8} c_{3 m} e^{2 x_{m}}+\frac{1}{2} c_{3 m}+\left(-\frac{7}{4} c_{3 m}+4 \kappa_{m 1}+\frac{3}{2} c_{3 m} x_{m}\right) e^{-2 x_{m}} \\
& +\left(\frac{11}{4} c_{3 m}-8 \kappa_{m 1}-3 c_{3 m} x_{m}\right) e^{-4 x_{m}}+O\left(e^{-6 x_{m}}\right),
\end{aligned}
$$

requires also $c_{3 m}=0$. Hence, denoting by $u_{m 1}^{(e)}$ the solution that has been exponentially matched to the inner solution $u_{0}$ near $x=0$, we obtain

$$
u_{m 1}^{(e)}\left(x_{m}\right)=\left(1-\tanh ^{2}\left(x_{m}\right)\right) \kappa_{m 1} .
$$

Internal layer near $x=0 \quad$ The $O\left(\delta^{1 / 3}\right)$ problem is

$$
\begin{aligned}
& \mathcal{L}\left(u_{01}, x_{0}\right)=f_{01}\left(x_{0}\right), \\
& u_{01}(0)=0, \quad u_{01}^{\prime \prime}(0)=0 \quad \text { and } \quad u_{01}^{\prime \prime \prime \prime}(0)=0,
\end{aligned}
$$

with

$$
f_{01}\left(x_{0}\right):=c_{10} x_{0}^{2}+c_{20} x_{0}+c_{30} .
$$

Its general solution reads

$$
u_{01}\left(x_{0}\right)=\varphi_{01}\left(x_{0}\right)+g_{1} \psi_{0}\left(x_{0}\right),
$$


where the homogeneous solutions are as before and the inhomogeneous solution is given by equation (3.21) with $\alpha=0, j=1$ and

$$
\gamma_{0}=-\frac{\pi^{2}}{12} c_{10}+\ln (2) c_{20}-c_{30}
$$

so that $\varphi_{01}(0)=0$ and $\varphi_{01}$ grows algebraically as $x_{0} \rightarrow-\infty$. Furthermore, symmetry requires $\varphi_{01}^{\prime \prime}(0)=0$ and $\varphi_{01}^{\prime \prime \prime \prime}(0)=0$, which implies $c_{10}=0$ and $c_{30}=0$ leading to

$$
\begin{aligned}
\varphi_{01}\left(x_{0}\right)= & \frac{c_{20}}{16\left(1+e^{-2 x_{0}}\right)^{2}}\left(1-4 x_{0}+12 \operatorname{dilog}\left(e^{2 x_{0}}+1\right) e^{-2 x_{0}}-e^{-4 x_{0}}+12 x_{0}^{2} e^{-2 x_{0}}\right. \\
& +\pi^{2} e^{-2 x_{0}}+12 x_{0} e^{-4 x_{0}}-14 x_{0} e^{-2 x_{0}}-\ln \left(1+e^{-2 x_{0}}\right) e^{2 x_{0}}+8 e^{-4 x_{0}} \ln \left(1+e^{-2 x_{0}}\right) \\
& \left.\left.-8 \ln \left(1+e^{-2 x_{0}}\right)\right)+e^{-6 x_{0}} \ln \left(1+e^{-2 x_{0}}\right)+2 e^{-6 x_{0}} x_{0}\right)
\end{aligned}
$$

where dilog denotes the dilogarithm function. The remaining free parameters of $u_{01}$ to be matched are $c_{20}$ and $g_{1}$. As will be demonstrated later, exponential matching to $u_{m}$ requires an expression for $u_{01}$ as $x_{0} \rightarrow-\infty$

$$
\begin{aligned}
u_{01}\left(x_{0}\right)= & -\frac{g_{1}}{16} e^{-2 x_{0}}-\frac{1}{4} c_{20} x_{0}-\frac{3}{8} g_{1} \\
& +\frac{1}{32}\left(2 c_{20} \pi^{2}+15 c_{20}+26 g_{1}+\left(48 g_{1}-12 c_{20}\right) x_{0}+24 c_{20} x_{0}^{2}\right) e^{2 x_{0}} \\
& +\frac{1}{48}\left(-36 g_{1}-89 c_{20}-6 c_{20} \pi^{2}+\left(84 c_{20}-144 g_{1}\right) x_{0}-72 c_{20} x_{0}^{2}\right) e^{4 x_{0}}+O\left(e^{6 x_{0}}\right)
\end{aligned}
$$

and then re-expanding $u_{0}$ in the variable $x_{m}$. This shows that also $c_{20}=0$, $g_{1}=0$ and $c_{3 m}=0$. Any other choice leads to a system for the parameters having no solution. Hence, only $\kappa_{m}$ remains as a free constant in the two regions. The exponentially matched solution is therefore simply

$$
u_{01}^{(e)}\left(x_{0}\right)=0 \text {. }
$$

\subsection{3 $\mathrm{O}\left(\delta^{2 / 3}\right)$}

Internal layer near $\kappa_{m}$ The problem of order $\delta^{2 / 3}$ is

$$
\begin{aligned}
& \mathcal{L}\left(u_{m 2}, x_{m}\right)=f_{m 2}\left(x_{m}\right), \\
& u_{m 2}(0)=-u_{m 0}^{\prime}(0) \kappa_{m 2}-\frac{1}{2} u_{m 0}^{\prime \prime} \kappa_{m 1}^{2}-u_{m 1}^{\prime}(0) \kappa_{m 1}=\kappa_{m 2}-u_{m 1}^{\prime}(0) \kappa_{m 1},
\end{aligned}
$$

where

$$
f_{m 2}\left(x_{m}\right):=d_{1 m} x_{m}^{2}+d_{2 m} x_{m}+d_{3 m}+6 u_{m 0}\left(u_{m 1}^{(e)}\right)^{2} .
$$

Note that $u_{m 1}^{(m)^{\prime}}(0)=0$. Again we choose the inhomogeneous solution so that it grows only algebraically as $x_{m} \rightarrow-\infty$ and vanishes at $x_{m}=0$ to obtain (3.21) with $\alpha=m, j=2$ and

$$
\gamma_{m 2}=-\frac{\pi^{2}}{12} d_{1 m}+\ln (2) d_{2 m}-d_{3 m}-\kappa_{m 1}^{2}
$$


so that the general solution is represented as

$$
u_{m 2}\left(x_{m}\right)=-\kappa_{m 2} \phi_{m}\left(x_{m}\right)+\varphi_{m 2}\left(x_{m}\right) .
$$

As $x_{m} \rightarrow-\infty$ we have to compare

$$
\begin{aligned}
u_{m 2}\left(x_{m}\right)= & -\frac{1}{8}\left(d_{1 m}+2 d_{3 m}\right)-\frac{1}{4} d_{2 m} x_{m}-\frac{1}{4} d_{1 m} x_{m}^{2} \\
& +e^{2 x_{m}}\left(\frac{1}{64}\left[(-7-72 \zeta(3)) d_{1 m}-8 d_{3 m}+256\left(\kappa_{m 2}-\kappa_{m 1}^{2}\right)+\left(30+4 \pi^{2}\right) d_{2 m}\right]\right. \\
& \left.+\frac{3}{16}\left(5 d_{1 m}-2 d_{2 m}+8 d_{3 m}\right) x_{m}+\frac{3}{8}\left(2 d_{2 m}-d_{1 m}\right) x_{m}^{2}+\frac{1}{2} d_{1 m} x_{m}^{3}\right)+O\left(e^{4 x_{m}}\right)
\end{aligned}
$$

with the outer solution. Matching the constant and the linear terms in $x_{m}$ yields

$$
\begin{gathered}
-\frac{1}{4} d_{3 m}=\frac{1}{2} A_{1}-\frac{1}{8} A_{1}^{2}+\frac{1}{3} C_{1} A_{1}+\frac{23}{14} C_{1}^{2}+D_{1} \\
-\frac{1}{4} d_{2 m}=2^{1 / 6} C_{1} .
\end{gathered}
$$

There is no quadratic term in the outer solution A.10), hence $d_{1 m}=0$. There are further matching conditions but they do not simplify the problem structurally at this point and will be enforced later, so that $d_{2 m}, d_{3 m}$ and $\kappa_{m 2}$ remain to be determined via exponential matching. As $x_{m} \rightarrow \infty$, the expansion to this order can be written as

$$
\begin{aligned}
& u_{m 2}^{(m)}=\frac{1}{2} d_{3 m}-\frac{1}{4} d_{2 m} x_{m}+\frac{1}{8} d_{3 m} e^{2 x_{m}}+\frac{e^{-2 x_{m}}}{32}\left(-56 d_{3 m}-15 d_{2 m}\right. \\
& \left.-2 d_{2 m} \pi^{2}+128\left(\kappa_{m 1}^{2}+\kappa_{m 2}\right)+\left(48 d_{3 m}-12 d_{2 m}\right) x_{m}-24 d_{2 m} x_{m}^{2}\right)+O\left(e^{-4 x_{m}}\right) .
\end{aligned}
$$

Internal layer near $x=0 \quad$ As for the $O\left(\delta^{1 / 3}\right)$ problem, at $O\left(\delta^{2 / 3}\right)$ we have

$$
\begin{aligned}
& \mathcal{L}\left(u_{02}, x_{0}\right)=f_{02}\left(x_{0}\right), \\
& u_{02}(0)=0, \quad u_{02}^{\prime \prime}(0)=0 \quad \text { and } \quad u_{02}^{\prime \prime \prime \prime}(0)=0
\end{aligned}
$$

with

$$
f_{02}\left(x_{0}\right):=d_{10} x_{0}^{2}+d_{20} x_{0}+d_{30} .
$$

The general solution is

$$
u_{02}\left(x_{0}\right)=\varphi_{02}\left(x_{0}\right)+g_{2} \psi_{0}\left(x_{0}\right),
$$

where the homogeneous component is as before and the inhomogeneous part is obtained by setting $\alpha=0, j=2$ and $\gamma_{02}=0$ in (3.21), so that $\varphi_{02}(0)=0$ and $\varphi_{02}$ grows algebraically as $x_{0} \rightarrow-\infty$. Symmetry requires $\varphi_{02}^{\prime \prime}(0)=0$, $\varphi_{02}^{\prime \prime \prime \prime}(0)=0$, which implies $d_{10}=0$ and $d_{30}=0$. The remaining free parameters 
to be matched are $d_{20}$ and $g_{2}$. In order to exponentially match to $u_{m}$ to $O\left(\delta^{2 / 3}\right)$ and obtain $u_{m 2}^{(e)}$, we again have to expand $u_{02}\left(x_{0}\right)$ as $x_{0} \rightarrow-\infty$, giving

$$
\begin{aligned}
u_{02}\left(x_{0}\right)= & -\frac{\hat{\mu}}{16} e^{-2 x_{0}}-\frac{1}{4} d_{20} x_{0}-\frac{3}{8} \hat{\mu} \\
& +\frac{1}{32}\left(\left(15+2 \pi^{2}+2 \ln (2)\right) d_{20}+26 g_{2}+\left(48 \hat{\mu}-12 d_{20}\right) x_{0}+24 d_{20} x_{0}^{2}\right) e^{2 x_{0}} \\
& +\frac{1}{48}\left(-\left(89+6 \pi^{2}\right) d_{20}-36 \hat{\mu}+\left(84 d_{20}-144 \hat{\mu}\right) x_{0}-72 d_{20} x_{0}^{2}\right) e^{4 x_{0}}+O\left(e^{6 x_{0}}\right),
\end{aligned}
$$

and re-express in terms of $x_{m}$, where we have used the abbreviation $\hat{\mu}=$ $d_{20} \ln (2)+g_{2}$.

\subsection{4 $\mathrm{O}(\delta)$}

Internal layer near $\kappa_{m}$ The problem to be solved at order $O(\delta)$ is

$$
\begin{aligned}
\mathcal{L}\left(u_{m 3}, x_{m}\right)= & f_{m 3}\left(x_{m}\right) \\
u_{m 3}(0)= & -u_{m 2}^{\prime}(0) \kappa_{m 1}-u_{m 0}^{\prime \prime}(0) \kappa_{m 1} \kappa_{m 2}-u_{m 0}^{\prime}(0) \kappa_{m 3} \\
& -\frac{1}{6} u_{m 0}^{\prime \prime \prime}(0) \kappa_{m 1}^{3}-u_{m 1}^{\prime}(0) \kappa_{m 2}-\frac{1}{2} u_{m 1}^{\prime \prime}(0) \kappa_{m 2}^{2},
\end{aligned}
$$

with

$$
\begin{aligned}
& f_{m 3}\left(x_{m}\right):=2\left(\left(u_{m 1}^{(e)}\right)^{3}+6 u_{m 0} u_{m 1}^{(e)} u_{m 2}^{(e)}\right) \\
& -2^{3 / 2}\left[\frac{1}{2} \operatorname{dilog}\left(e^{2 x_{m}}+1\right)+\frac{1}{2}\left(1+k_{1 m}\right) x_{m}^{2}+\left(\ln (2)+k_{2 m}\right) x_{m}+k_{3 m}\right] .
\end{aligned}
$$

Again we choose the inhomogeneous solution so that it grows only algebraically as $x_{m} \rightarrow-\infty$ and vanishes at $x_{m}=0$ and so that we obtain $\varphi_{m 3}\left(x_{m}\right)$ by using formula (3.21) with $\alpha=m, j=3$ and $\gamma_{m 3}=0$. The solution is

$$
u_{m 3}\left(x_{m}\right)=-u_{m 3}(0) \phi_{m}\left(x_{m}\right)+\varphi_{m 3}\left(x_{m}\right),
$$

where $k_{1 m}, k_{2 m}, k_{3 m}$ and $\kappa_{m 3}$ remain to be determined via matching. In order to exclude exponential growth as $x_{m} \rightarrow-\infty$ we obtain the relation

$$
\begin{aligned}
k_{2 m}= & \frac{\sqrt{2}}{48 \ln (2)}\left(\kappa_{m 1}\left(-\left(12+9 \pi^{2}\right) d_{2 m}+12 d_{3 m}-24 \kappa_{m 2}\right)\right. \\
& \left.+\sqrt{2}\left(24 k_{3 m}-12 \ln (2)^{2}+k_{1 m} \pi^{2}\right)\right)
\end{aligned}
$$

so that the expansion obtained as $x_{m} \rightarrow-\infty$ is

$$
\begin{aligned}
u_{m 3}\left(x_{m}\right)= & \frac{1}{4 \sqrt{2}}\left(1+k_{1 m}+4 k_{3 m}\right)+\frac{1}{\sqrt{2}}\left(\ln (2)+k_{2 m}\right) x_{m} \\
& +\left(k_{1 m}+1\right) \frac{\sqrt{2}}{4} x_{m}^{2}+O\left(e^{2 x_{m}}\right) .
\end{aligned}
$$


Comparing this with the outer solution to $O(\delta)$, equation (A.10), yields the matching conditions

$$
\begin{gathered}
\frac{1}{4 \sqrt{2}}\left(1+k_{1 m}+4 k_{3 m}\right)=\left(-\frac{1}{4} A_{1}+\frac{1}{3} C_{1}\right) A_{2}+\left(\frac{7}{12} C_{1}^{2}+\frac{1}{3} D_{1}\right) A_{1} \\
+\frac{1}{2} A_{3}-\frac{59}{216} C_{1} A_{1}^{2}-\frac{1}{12} 2^{1 / 3} C_{1}+K_{1}-\frac{23}{7} C_{1} D_{1}+\frac{1}{16} A_{1}^{3}+\frac{127}{28} C_{1}^{3}
\end{gathered}
$$

for the constant terms,

$$
\frac{1}{\sqrt{2}}\left(\ln (2)+k_{2 m}\right)=\left(D_{1}-\frac{23}{7} C_{1}^{2}\right) 2^{1 / 6} \quad \text { and } \quad\left(k_{1 m}+1\right) \frac{\sqrt{2}}{4}=2^{-2 / 3} C_{1}
$$

for the linear and the quadratic terms, respectively.

Expanding the solution as $x_{m} \rightarrow \infty$ we find

$$
\begin{aligned}
u_{m 3}\left(x_{m}\right)= & \frac{1}{192}\left(\kappa_{m 1} d_{2 m}\left(9 \pi^{2}+24\right)-48 \kappa_{m 1} d_{3 m}+2 \sqrt{2} \pi^{2}\left(1-k_{1 m}\right)-48 \sqrt{2} k_{3 m}\right) e^{2 x_{m}} \\
& +\frac{1}{96}\left(\kappa_{m 1} d_{2 m}\left(27 \pi^{2}+72\right)+\sqrt{2}\left(k_{1 m}\left(12-6 \pi^{2}\right)-96 k_{3 m}-12+2 \pi^{2}\right)\right) \\
& +\frac{1}{\sqrt{2}}\left(\ln (2)+k_{2 m}\right) x_{m}+\left(k_{1 m}+1\right) \frac{\sqrt{2}}{4} x_{m}^{2}+O\left(e^{-2 x_{m}}\right),
\end{aligned}
$$

and we will exponentially match it to the solution near $x=0$, which we construct next.

Internal layer near $x=0$ The general solution to the $O(\delta)$ problem

$$
\begin{aligned}
& \mathcal{L}\left(u_{03}, x_{0}\right)=f_{03}\left(x_{0}\right), \\
& u_{03}(0)=0, \quad u_{03}^{\prime \prime}(0)=0 \quad \text { and } \quad u_{03}^{\prime \prime \prime \prime}(0)=0,
\end{aligned}
$$

with

$$
f_{03}\left(x_{0}\right):=-2^{1 / 2}\left[\operatorname{dilog}\left(e^{2 x_{0}}+1\right)-\operatorname{dilog}(2)+2 \mu_{2} x_{0}+\left(1+k_{10}\right) x_{0}^{2}\right]
$$

and the abbreviation $\mu_{2}=\ln (2)+k_{20}$ reads

$$
u_{03}\left(x_{0}\right)=\varphi_{03}\left(x_{0}\right)+g_{3} \psi_{0}\left(x_{0}\right),
$$

where we have required that $u_{03}(0)=0$ and $u_{03}^{\prime \prime}(0)=0$. If we also enforce $u_{03}^{\prime \prime \prime}(0)=0$ then $k_{10}=0$. Again we take an inhomogeneous solution $\varphi_{03}\left(x_{0}\right)$ which satisfies the above conditions, so that the general solution is obtained with

$\mu_{1}=\sqrt{2}\left(\ln (2)^{2}+2 k_{20} \ln (2)\right)-g_{3} \quad$ and $\quad \omega=\int_{0}^{1} \frac{1}{z} \ln \left(\frac{z^{2}+1}{2 z}\right)^{2}-\frac{\ln (2 z)^{2}}{z} d z \approx 0.3094$, 


$$
\begin{aligned}
u_{03}= & \frac{12 \mu_{1}-\pi^{2} \sqrt{2}}{192} e^{-2 x_{0}}+\frac{1}{96}\left(36 \mu_{1}+\sqrt{2}\left(12-\pi^{2}\right)\right)+\frac{\mu_{2}}{\sqrt{2}} x_{0}+\frac{\sqrt{2}}{4} x_{0}^{2} \\
& +\left[\frac{1}{192}\left(156 \mu_{1}+\sqrt{2}\left[\left(19-24 k_{20}\right) \pi^{2}-15-288 \omega-180 \mu_{2}\right]\right)\right. \\
& \left.+\frac{1}{16}\left(-24 \mu_{1}+\sqrt{2}\left(12 \mu_{2}-11\right)\right) x_{0}+\frac{\sqrt{2}}{8}\left(3-12 \mu_{2}\right) x_{0}^{2}-\frac{1}{\sqrt{2}} x_{0}^{3}\right] e^{2 x_{0}}+O\left(e^{4 x_{0}}\right) .
\end{aligned}
$$

For exponentially matching to $u_{m}$ this again has to be re-expressed in $x_{m}$ and combined with the corresponding expressions for $u_{00}, u_{01}$ and $u_{02}$. This will be done in the next section.

\subsection{Exponential matching}

Now we have to match the rest of the solution $u_{m}\left(x_{m}\right)$ to the rest of the solution $u_{0}\left(x_{0}\right)$. This requires matching the exponential terms in addition to the algebraic terms, similarly to the procedure for the $\mathrm{CCH}$ equation, i.e. matching of the solution describing the internal layer near $x=\kappa_{m}$ to the solution near $x=0$ requires expressing the variable $x_{0}$ in terms of $x_{m}$ (or vice versa). Recall again that $x_{0}=x_{m}+\bar{\kappa}_{m} / \sqrt{2}$ and that $\bar{\kappa}_{m}<0$; the $e^{2 x_{0}}$ terms in the $u_{0}$ expansion will produce $e^{2 x_{m}}$ terms with a factor $e^{\sqrt{2} \bar{\kappa}_{m}}$ (and analogously for $e^{-2 x_{0}}$ terms) and so we will find their corresponding matching partner at a different order in $\delta$ in the $u_{m}$ expansion, as we have shown for the $\mathrm{CCH}$ equation. The somewhat subtle difference here is that additionally we need to determine the relationship between $e^{\sqrt{2} \bar{\kappa}_{m}}$ and $\delta$ and we have in principle several choices, only one of which allows a consistent matching of both expansions. One can observe that the choice $e^{\sqrt{2} \bar{\kappa}_{m}}=\rho \delta^{1 / 3}$, where $\rho$ is some constant quickly leads to a contradiction. However, setting

$$
e^{\sqrt{2} \bar{\kappa}_{m}}=\rho \delta^{2 / 3}
$$

will lead to a $O\left(\delta^{2 / 3}\right)$ shift of terms, so that e.g.

$$
\begin{array}{cc}
e^{2 x_{0}} & \text { will shift to a term } \quad \delta^{2 / 3} e^{2 x_{m}}, \\
e^{-2 x_{0}} & \text { will end up as a term } \delta^{-2 / 3} e^{-2 x_{m}}
\end{array}
$$

and so forth, so that e.g. a term $e^{2 x_{0}}$ in the leading order part of the $u_{0}$ expansion will have to match a $e^{2 x_{m}}$ term in the $O\left(\delta^{2 / 3}\right)$ part of the $u_{m}$ expansion, or a $e^{-2 x_{0}}$ term in the $O(\delta)$ part of the $u_{0}$ expansion will have to match a $e^{-2 x_{m}}$ term in the $O\left(\delta^{1 / 3}\right)$ part of the $u_{m}$ expansion. This will also produce terms that will have no partner term in the transformed expansion. Their coefficients must then be set to zero. If we now sum the expansions for $u_{01}\left(x_{0}\right), u_{02}\left(x_{0}\right)$ 
and $u_{03}\left(x_{0}\right)$ and re-expand using (3.57), we obtain

$$
\begin{aligned}
u_{0}\left(x_{m}\right)= & -1-\frac{1}{16}\left(d_{20} \ln (2)+g_{2}\right) e^{-2 x_{m}} \rho+\frac{1}{192}\left(12 \mu_{1}-\sqrt{2} \pi^{2}\right) e^{-2 x_{m}} \rho \delta^{1 / 3} \\
& +\frac{1}{24}\left(d_{20}(3 \ln (\rho)-9 \ln (2)-2 \ln (\delta))-9 g_{2}-6 d_{20} x_{m}+48 e^{2 x_{m}} / \rho\right) \delta^{2 / 3} \\
& +\left[\frac{1}{96}\left(36 \mu_{1}+\sqrt{2}\left[12+(16 \ln (\delta)-24 \ln (\rho)) \mu_{2}+6\left(\ln (\rho)-\frac{2}{3} \ln (\delta)\right)^{2}-\pi^{2}\right]\right)\right. \\
& \left.+\frac{\sqrt{2}}{12}\left(2 \ln (\delta)+6 \mu_{2}-3 \ln (\rho)\right) x_{m}+\frac{\sqrt{2}}{4} x_{m}^{2}\right] \delta,
\end{aligned}
$$

which has to match $u_{m 1}\left(x_{m}\right), u_{m 2}\left(x_{m}\right)$ and $u_{m 3}\left(x_{m}\right)$ to each order, respectively. From this we obtain further conditions for the parameters in addition to those we have already found. Solving the complete system of equations then yields the solutions for the width of the hump

$$
\Delta=\frac{\sqrt{2}}{6} \ln \left(\frac{\beta}{W\left(\beta^{1 / 3}\right)^{3}}\right),
$$

with $\beta=2^{11} /\left(27 \delta^{2}\right)$, where $W$ is the Lambert $W$ function (so $W(x)$ is the solution of $x=W \exp (W))$. The expressions for the remaining matching constants $C_{1}, D_{1}$, etc. are omitted. The first correction in (3.2) has the coefficient

$$
A_{1}=-32^{1 / 6} \text {. }
$$

Note that in the transformed expansions as well as in the expressions for the parameters also contain so-called logarithmic switch-back terms.

\section{Numerical method for the fifth-order phase space}

For the numerical stationary solutions of the $\mathrm{HCCH}$ equation 3.1 we apply the same scaling for $u$ that we used for the $\mathrm{CCH}$ equation to obtain equilibrium points at \pm 1 .

$$
\left(1-c^{2}\right)=\frac{2}{\delta \sqrt{A}}\left(c_{x x}+c-A c^{3}\right)_{x x x}, \quad \lim _{x \rightarrow \pm \infty} c=\mp 1,
$$

again assuming that derivatives vanish in the far field. Reduction to a first order system $U^{\prime}=F(U)$, with $F: \mathbb{R}^{5} \rightarrow \mathbb{R}^{5}$, gives a five-dimensional phase space, where the first four components of $F_{i}(U)$ are equal to $U_{i+1}$ and the fifth is

$$
F_{5}(U)=6 A\left(U_{2}\right)^{3}+18 A U_{1} U_{2} U_{3}+\left(3 A\left(U_{1}\right)^{2}-1\right) U_{4}+\delta \sqrt{A}\left(1-\left(U_{1}\right)^{2}\right) / 2 .
$$

The equilibrium points are $U^{ \pm}= \pm(1,0,0,0,0)^{T}$ and at these points the characteristic polynomials are

$$
\mathcal{P}^{ \pm}(\lambda)=\lambda^{5}+\lambda^{3}(1-3 A) \pm \delta \sqrt{A} .
$$


For small $\delta$ the manifolds $W^{u}\left(U^{+}\right)$and $W^{s}\left(U^{-}\right)$are both two-dimensional, resulting in a codimension two event when searching for heteroclinic solutions connecting the two hyperbolic fixed points $U^{+}$and $U^{-}$. The $\mathrm{HCCH}$ equation exhibits the same reversibility properties as its lower order version. This reversibility is again given by the transformation (2.8) from the $\mathrm{CCH}$ section, which also here fulfills $R F(U)=-F(R U)$. The codimension reduces by one and again we deal with a codimension one problem and two parameters, hence we may expect solution branches in the $(A, \delta)$ parameter plane. Section 2.1 showed that a condition for the existence of heteroclinic orbits is a value where the distance function (2.9) reaches zero and the same condition holds for the $\mathrm{HCCH}$ equation. The phase space is sketched in figure [6 indicating the linearizations of the intersecting manifolds in the equilibrium points. For this

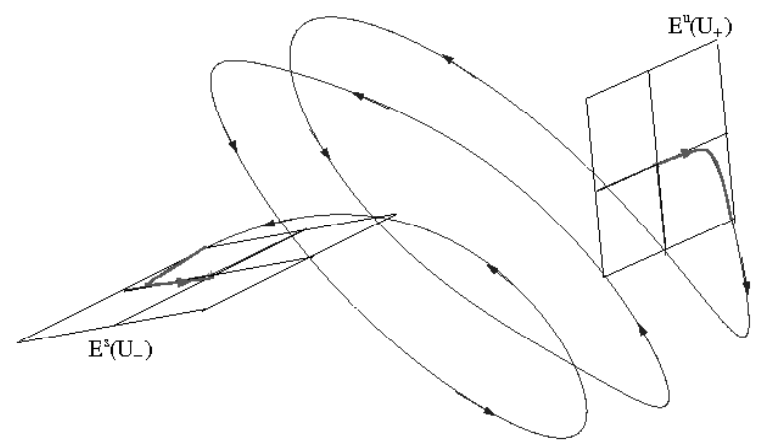

Figure 6: HCCH: Heteroclinic orbits between the equilibrium points are sought in a 5 -D phase space that is indicated here in $3 \mathrm{D}$. The manifolds $W^{u}\left(U^{+}\right)$ and $W^{s}\left(U^{-}\right)$are two-dimensional which is suggested by the two planes in the picture.

problem a shooting method will be very slow and may lead to bad accuracy since the additional parameter, say $\varphi \in[0,2 \pi)$, an angle defining points on a circle close to the equilibrium point on the linearization of the two-dimensional manifold, requires a very fine resolution to obtain heteroclinic solutions.

\subsection{Boundary value problem formulation}

There exist several possibilities to set up equations for finding heteroclinic connections in a boundary value problem framework. Generally one crucial stumbling block is the choice of a suitable phase condition that picks a certain solution out of the infinitely many available ones due to phase shifts [2, 8]. We choose to incorporate one phase condition proposed by Beyn [2], for which we use an approximation of the solution, $V$, typically given by a previous solution for slightly different parameter values. Equation (4.1) contains two parameters, $A, \delta$, and in addition the truncated domain length $L$. As discussed by Doedel et al. [5] one of the free parameters can be replaced by $L$ to find a connection. 
We replace $\delta$, solve and continue after extrapolating to an approximate value of $A$ for a nearby chosen and fixed $\delta$. Rescaling the domain to $[0,1]$ yields, with the phase condition variable $U_{p h}$ introduced by Beyn [2] the first order system

$$
\begin{aligned}
U_{i}^{\prime} & =L U_{i+1}, \quad i=1,2,3,4 \\
U_{5}^{\prime} & =L\left(6 A\left(U_{2}\right)^{3}+18 A U_{1} U_{2} U_{3}+\left(3 A\left(U_{1}\right)^{2}-1\right) U_{4}+\delta \sqrt{A} \frac{\left(1-\left(U_{1}\right)^{2}\right)}{2}\right) \\
U_{p h}^{\prime} & =L\left(V^{\prime}\right)^{T} U \\
L^{\prime} & =0, A^{\prime}=0
\end{aligned}
$$

Hence, we obtain one equation for the phase condition and two for the parameters in addition to the five given by the original ODE, i.e., we have an overall system of eight equations which have to be supplemented by the same number of boundary conditions. At the edges of the domain we utilize projected boundary conditions 2, 4, ], which make use of eigenvectors in the equilibrium points and can be incorporated by computing $V_{0}$, the matrix whose columns are composed by the eigenvectors which correspond to the eigenvalues at the upper equilibrium point $U^{+}$with negative real part, and by forming the counterpart $V_{1}$ containing those eigenvectors given by the unstable directions at the lower stationary point $U^{-}$. Hence, we consider the eight boundary conditions

$$
U_{p h}(0)=0, \quad U_{p h}(1)=0, \quad V_{0}^{T}\left(U(0)-U^{+}\right)=0, \quad V_{1}^{T}\left(U(1)-U^{-}\right)=0 .
$$

For initial estimates we can use solutions obtained from the asymptotic analysis of section 3.1 i.e. the leading order solution tanh profiles

$$
V(x)=-\tanh (x-K)+\tanh (x)-\tanh (x+K),
$$

for the het $t_{1}$ solution with guessed root-distance $K$.

The boundary value solvers we use are based on mono-implicit Runge-Kutta formulae [14, 23]. As for the CCH problem efficiency can be improved by making use of the theory from section 2.1 which holds analogously for the $\mathrm{HCCH}$ equation to obtain a boundary condition at the fixed point of a point-symmetric solution. We can use half of the previous domain length and phase conditions become redundant, because the phase is already fixed. We replace the projected boundary conditions by

$$
U_{1}(0)=1, \quad U_{2}(0)^{2}+U_{3}(0)^{2}=0, \quad U_{4}(0)^{2}+U_{5}(0)^{2}=0
$$

so that together with the self-reversibility condition on the right interval end $U_{1}(1)=U_{3}(1)=U_{5}(1)=0$ we have six conditions which match the five equations together with the free parameter $A$. Final solutions are obtained by reflecting the solution and its derivatives around zero and changing the signs of the first, third and fifth component. Examples of branches of different solutions are shown in figure 7 . 

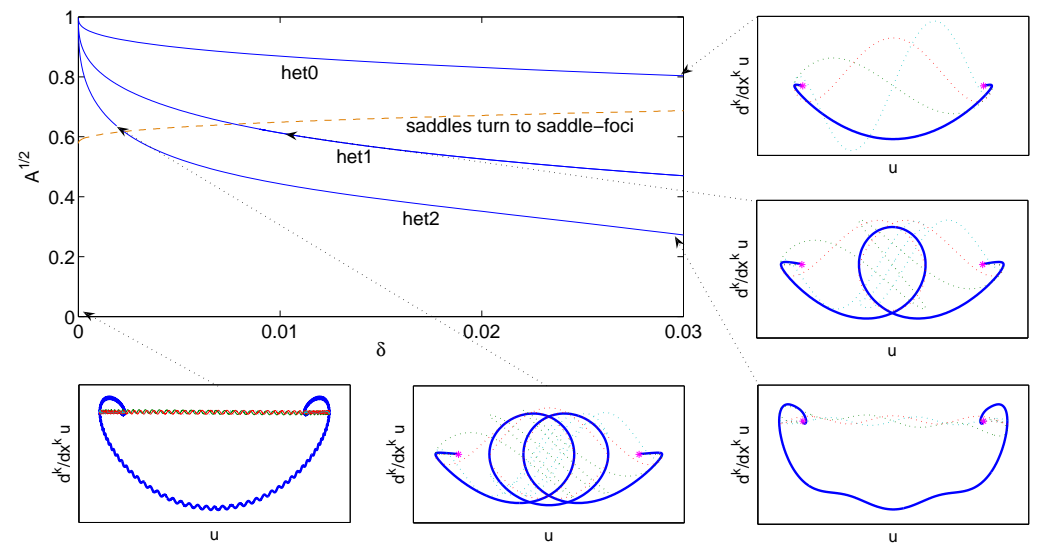

Figure 7: $(\sqrt{A}, \delta)$ - plane with curves for the first three heteroclinic connection branches for the $\mathrm{HCCH}$ equation. The dashed line in the parameter plane indicates the position where the positive roots of the characteristic polynomial in $U^{+}$have nonzero imaginary parts. Below and to the right we see five phase space diagrams (tuples $\left(U_{1}, U_{2}\right),\left(U_{1}, U_{3}\right), \ldots$ ) for selected solutions pointed out with arrows marking the corresponding parameters. The first pair $\left(U_{1}, U_{2}\right)$ is plotted as bold solid curve.

\subsection{Solutions and comparison to analytical results}

With the boundary value formulation we are able to compute new HCCH stationary solutions. In figure 8 we see a particular het s $_{2}$ solution and the profile of the growing structure.
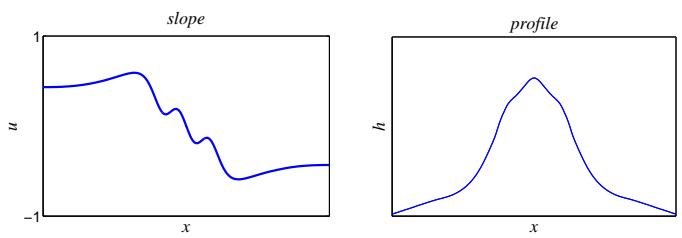

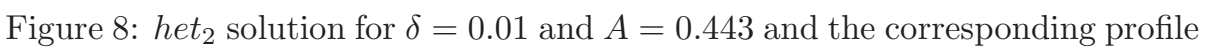
obtained by integration.

Up to 3D one can nicely visualize heteroclinic orbits in the corresponding phase space, while when the dimension is four or higher and the derivatives vanish in the far field one can still plot the $2 \mathrm{D}$ phase spaces $\left(U_{1}, U_{2}\right),\left(U_{1}, U_{3}\right), \ldots$ and demand connections between the equilibrium tuples $( \pm \sqrt{A}, 0)$ as a necessary condition for heteroclinic orbits in the higher order space. Several such projections onto $2 \mathrm{D}$ are shown in figure 7 where we also see a very rapidly oscillating heteroclinic curve in the bottom left plot which was found by a shooting 
approach with a minimization procedure that used the two parameters and an angle as free parameters and the distance function (2.9) as objective function, depending on those parameters. It indicates that as shown for the $\mathrm{CCH}$ equation we can in fact find many more het $_{k}$ branches than those presented for $k=0,1,2$, all emerging from $(A, \delta)=(1,0)$, which corresponds to the CahnHilliard equation.

In figure 9 we see the change in appearance of solutions on the het $_{2}$ branch as $\delta$ is increased. The shape varies from a solution with two pronounced humps to a monotone one, similar to the het $_{0}$ solution, although associated with different, smaller, values of $A$. This is crucial if one wants to compute solutions for bigger $\delta$ with a boundary value solver. It easily happens that the solver switches between solution branches, however, this can be prevented by starting continuation in a parameter regime where the high-slope parts of the solutions are non-monotone, and continuing with small steps. A characteristic of the $\mathrm{HCCH}$ solutions is the
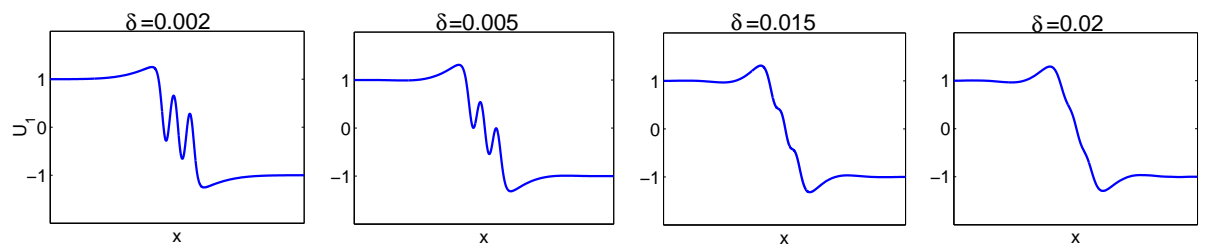

Figure 9: Structural change of the scaled het s $_{2}$ solution as $\delta$ is increased.

overshoot from the equilibrium value before the solutions go down. This is not observed for the $\mathrm{CCH}$ equation, where the shape is similar at these regions to hyperbolic tangent functions.

In light of the expansion (3.2) we try to estimate the $\mathcal{O}\left(\delta^{1 / 3}\right)$ terms $A_{1}$ for the different heteroclinic connections in a range of very small $\delta$. As we see in figure 10 on the left, the numerically obtained values for $A$ behave like $A=1-2^{1 / 6} \delta^{1 / 3}$ in case of the het $t_{0}$ solutions, so that $A_{1}=-2^{1 / 6}$, which is consistent with the result in Savina et al. [22]. The numerical result for het t $_{1}$ is in line with the analytical value (3.62) and since for het $t_{2}$ we see the agreement $A_{1} \approx-52^{1 / 6}$, we propose for higher order trajectories that for het $t_{k}$ we have the general approximation $A_{1} \approx-(2 k+1) 2^{1 / 6}$, which is reminiscent of the CCH expression (2.45). Hence this formula is used in figure 10 to plot the analytical values.

We measure the distance between the first and second root for the het $t_{1}$ and the het $_{2}$ solutions as seen in figure 10 on the right. We compare this to the analytical expression (3.61) for the one-hump solutions in the same figure and see that for small $\delta$ the agreement is good. For both het h $_{1}$ and het $_{2}$ solutions the distance is seen to increase logarithmically as $\delta$ decreases. 

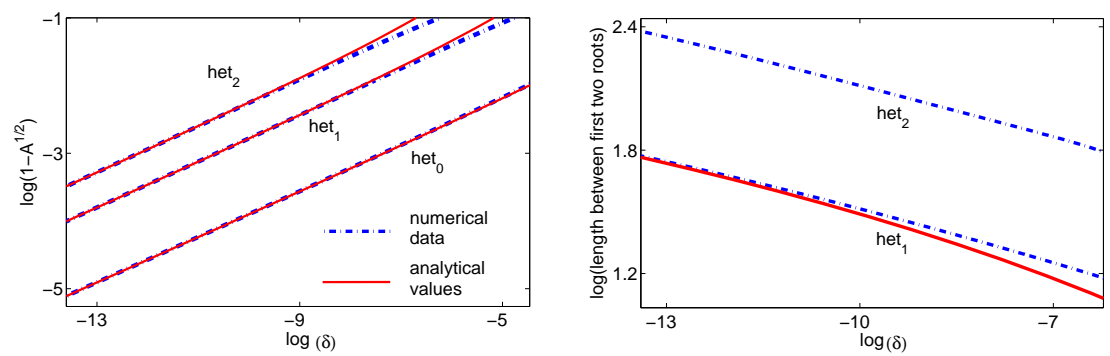

Figure 10: Left figure: Logarithmic version of the $(\sqrt{A}, \delta)$ plot for very small $\delta$. Drawn through curves giving the analytical values, dash-dotted lines those computed with the BVP solver. On the right we see the distances between the first two roots of the het ${ }_{1}$, het $t_{2}$ solutions, numerically and for het $t_{1}$ via the analytical expression (3.61) (solid line).

\section{Conclusion}

We have demonstrated that a sixth-order generalization of the convective CahnHilliard equation admits multiple stationary solutions connecting constant values. As for the fourth-order convective Cahn-Hilliard solution, these include a simple base solution, which is monotone for the $\mathrm{CCH}$ and "almost" monotone for the HCCH equation. More complex solutions, containing multiple humps, are also possible for each value of the forcing parameter $\delta$, given particular values of the integration constant $A(\delta)$. These non-monotone stationary solutions constitute an essential part of the solution structure for this higher-order Cahn-Hilliard type equation. We have demonstrated this via a numerical investigation of the phase space in which we are able to follow solution branches. For the simplest of the multi-humped solutions, the het $t_{1}$ branch, careful use of matched asymptotics that accounts for exponentially small terms allows us to find a solution which yields both the length scale for the solution (the "hump length") and the parameter value $A(\delta)$ at which it occurs, in the limit of small $\delta$. Extension of the analysis to higher branches appears feasible. Our numerical evidence suggests that similarly simple asymptotic expressions hold for these branches, for both the $\mathrm{CCH}$ and $\mathrm{HCCH}$ equations. Physically, these solutions may represent situations where the edge energy regularization represented in (1.4) fails to produce a smooth transition between facets.

Various issues, such as the stability of these solutions are presently being considered in the light of applications of the $\mathrm{HCCH}$ equation as a model for the morphology and dynamics of quantum dots. In particular, how do adjacent internal layers derived from these solutions interact, and what is their effect on the coarsening behavior in large spatial domains? Savina et al. 222] have begun an investigation of these questions by numerical simulation of (1.4); it is likely that asymptotics can yield further insights.

Physically, further interesting questions relate to the extension of the $\mathrm{HCCH}$ 
model to richer models for the energetics of facetted surfaces, and analyzing the three-dimensional extension of the model.

\section{Acknowledgments}

This work was performed as part of Project C-10 of the DFG research center Matheon, Berlin. AM also gratefully acknowledges the support from the Heisenberg Fellowship of the DFG (grant MU 1626/3).

\section{A Outer Problem}

For the solution to the outer problem (3.6), (3.7) it is easy to observe that to leading order in $\delta$ the solution of

$$
Q\left(Y_{0}\right)_{\xi \xi \xi}=-\sqrt{2}\left(Y_{0}^{2}-1\right) \quad \text { with } \lim _{\xi \rightarrow-\infty} Y_{0}(\xi)=1
$$

is

$$
Y_{0}(\xi)=1
$$

To $O\left(\delta^{1 / 3}\right)$ the general solution to the problem

$$
Y_{1_{\xi \xi \xi}}-\sqrt{2} Y_{1}=-\frac{A_{1}}{\sqrt{2}} \quad \text { with } \quad \lim _{\xi \rightarrow-\infty} Y_{1}(\xi)=\frac{A_{1}}{2}
$$

is

$$
Y_{1}(\xi)=\frac{A_{1}}{2}+C_{1} e^{2^{1 / 6} \xi}+e^{-\xi / 2^{5 / 6}}\left[C_{2} \cos \left(\sqrt{3} \xi / 2^{5 / 6}\right)+C_{3} \sin \left(\sqrt{3} \xi / 2^{5 / 6}\right)\right],
$$

with $C_{1}, C_{2}$ and $C_{3}$ being constants of integration. The far field condition requires that $Y_{1}$ remains bounded as $\xi \rightarrow-\infty$. Hence, $C_{2}=C_{3}=0$ and

$$
Y_{1}(\xi)=\frac{A_{1}}{2}+C_{1} e^{2^{1 / 6} \xi}
$$

Using this and the far field conditions, the solution to the $O\left(\delta^{2 / 3}\right)$ problem

$Y_{2_{\xi \xi \xi}}-\sqrt{2} Y_{2}=-\frac{A_{2}}{\sqrt{2}}-\frac{1}{2}\left(3\left(Y_{1}^{2}\right)_{\xi \xi \xi}-\sqrt{2} Y_{1}^{2}\right) \quad$ with $\quad \lim _{\xi \rightarrow-\infty} Y_{2}(\xi)=\frac{A_{2}}{2}-\frac{A_{1}^{2}}{8}$

is

$$
Y_{2}(\xi)=\frac{A_{2}}{2}-\frac{A_{1}^{2}}{8}+D_{1} e^{2^{1 / 6} \xi}+\frac{A_{1} C_{1}}{3} e^{2^{1 / 6} \xi}\left(1-2^{1 / 6} \xi\right)-\frac{23}{14} C_{1}^{2} e^{2^{7 / 6} \xi}
$$

and to the $O(\delta)$ problem

$$
\begin{aligned}
Y_{3 \xi \xi \xi}-\sqrt{2} Y_{3}=- & \frac{A_{2}}{\sqrt{2}}+\frac{Y_{1_{\xi \xi \xi \xi}}}{4}+\sqrt{2} Y_{1} Y_{2}-\frac{1}{2}\left(Y_{1}^{3}+6 Y_{1} Y_{2}\right)_{\xi \xi \xi} \\
& \text { with } \lim _{\xi \rightarrow-\infty} Y_{3}(\xi)=\frac{A_{3}}{2}-\frac{A_{1} A_{2}}{4}+\frac{A_{1}^{3}}{16}
\end{aligned}
$$


it is

$$
\begin{aligned}
Y_{3}(\xi)= & \frac{A_{3}}{2}-\frac{A_{1} A_{2}}{4}+\frac{A_{1}^{3}}{16} \\
& +\left[K_{1}-\frac{2^{1 / 3}}{12} C_{1}+\frac{1}{3}\left(A_{1} D_{1}+A_{2} C_{1}\right)-\frac{59}{216} C_{1} A_{1}^{2}\right. \\
& \left.+\left(\frac{\sqrt{2}}{12} C_{1}-\frac{2^{1 / 6}}{3}\left(A_{1} D_{1}+A_{2} C_{1}\right)+\frac{17}{72} 2^{1 / 6} A_{1}^{2} C_{1}\right) \xi+\frac{2^{1 / 3}}{18} A_{1}^{2} C_{1} \xi^{2}\right] e^{2^{1 / 6} \xi} \\
& +\left[-\frac{23}{7} C_{1} D_{1}+\left(\frac{7}{12}+\frac{23}{21} 2^{1 / 6} \xi\right) A_{1} C_{1}^{2}\right] e^{2^{7 / 6} \xi}+\frac{127}{28} C_{1}^{3} e^{2^{1 / 6} 3 \xi}
\end{aligned}
$$

with another integration constant $K_{1}$. Finally, we obtain the asymptotic representation in terms of $x_{m}$ :

$$
\begin{aligned}
Y\left(x_{m}\right)= & 1+\left[C_{1}+\frac{1}{2} A_{1}\right] \delta^{1 / 3}+\left[C_{1} 2^{1 / 6} x_{m}-\frac{1}{8} A_{1}^{2}+\frac{1}{3} C_{1} A_{1}+D_{1}-\frac{23}{14} C_{1}^{2}+\frac{1}{2} A_{2}\right] \delta^{2 / 3} \\
& +\left[-\frac{23}{7} C_{1}^{2} 2^{1 / 6} x_{m}+D_{1} 2^{1 / 6} x_{m}+\frac{1}{2} C_{1} 2^{1 / 3} x_{m}^{2}+\left(-\frac{1}{4} A_{1}+\frac{1}{3} C_{1}\right) A_{2}\right. \\
& +\left(\frac{7}{12} C_{1}^{2}+\frac{1}{3} D_{1}\right) A_{1}+\frac{1}{2} A_{3}-\frac{59}{216} C_{1} A_{1}^{2}-\frac{1}{12} 2^{1 / 3} C_{1} \\
& \left.+K_{1}-\frac{23}{7} C_{1} D_{1}+\frac{1}{16} A_{1}^{3}+\frac{127}{28} C_{1}^{3}\right] \delta .
\end{aligned}
$$

\section{References}

[1] K. L. Adams, J. R. King, and R. H. Tew. Beyond-all-orders effects in multiple-scales asymptotics: travelling-wave solutions to the KuramotoSivashinsky equation. J. Engr. Math., 45:197-226, 2003.

[2] W.-J. Beyn. The numerical computation of connecting orbits in dynamical systems. IMA Journal of Numerical Analysis, 9:379-405, 1990.

[3] G. Carrier and C. Pearson. Ordinary differential equations. Blaisdell, Waltham, Massachusetts, 1968.

[4] F. de Hoog and R. Weiss. An approximation theory for boundary value problems on infinite intervals. Computing, 24:227-239, 1980.

[5] E. Doedel and M. Friedman. Numerical computation of heteroclinic orbits. Journal of Computational and Applied Mathematics, 26:155-170, 1989.

[6] A. Eden and V. K. Kalantarov. The convective Cahn-Hilliard equation. Applied Mathematics Letters, 20(4):455-461, 2007. 
[7] C. L. Emmott and A. J. Bray. Coarsening dynamics of a one-dimensional driven Cahn-Hilliard system. Phys. Rev. E, 54:4568-4575, 1996.

[8] M. Friedman and E. Doedel. Numerical computation and continuation of invariant manifolds connecting fixed points. SIAM J. Num. Analysis, 28(3):789-808, 1991.

[9] A. A. Golovin, S. H. Davis, and A. A. Nepomnyashchy. A convective CahnHilliard model for the formation of facets and corners in crystal growth. Phys. D, 122(1-4):202-230, 1998.

[10] A. A. Golovin, A. A. Nepomnyashchy, S. H. Davis, and M. A. Zaks. Convective Cahn-Hilliard models: From coarsening to roughening. Phys. Rev. Lett., 86:1550-1553, 2001.

[11] M. E. Gurtin. Thermomechanics of Evolving Phase Boundaries in the Plane. Clarendon Press, Oxford, UK, 1993.

[12] C. J. Howls, T. Kawai, and Y. Takei, editors. Toward the exact WKB analysis of differential equations, linear or nonlinear. Kyoto University Press, Kyoto, 1999.

[13] W. Kath, C. Knessl, and B. Matkowsky. A variational approach to nonlinear singularly perturbed boundary value problems. Stud. Appl. Math., $77: 61-88,1987$.

[14] J. Kierzenka and L. Shampine. A BVP solver based on residual control and the MATLAB PSE. ACM Transactions on Mathematical Software, 27(3):299-316, Sept. 2001.

[15] C. G. Lange. On spurious solutions of singular perturbation problems. Stud. Appl. Math., 68:227-257, 1983.

[16] K.-T. Leung. Theory on morphological instability in driven systems. Journal of Statistical Physics, 61:345-364, 1990.

[17] F. Liu and H. Metiu. Dynamics of phase separation of crystal surfaces. Phys. Rev. B, 48:5808-5817, 1993.

[18] R. E. O'Malley, Jr. Phase-plane solutions to some singular perturbation problems. J. Math. Anal. Appl., 54(2):449-466, 1976.

[19] L. G. Reyna and M. J. Ward. Metastable internal layer dynamics for the viscous Cahn-Hilliard equation. Methods Appl. Anal., 2:285-306, 1995.

[20] S. Rosenblat and R. Szeto. Multiple solutions of nonlinear boundary-value problems. Stud. Appl. Math., 63:99-117, 1980.

[21] Y. Saito and M. Uwaha. Anisotropy effect on step morphology described by Kuramoto-Sivashinsky equation. J. Phys. Soc. Jpn., 65:3576-3581, 1996. 
[22] T. V. Savina, A. A. Golovin, S. H. Davis, A. A. Nepomnyashchy, and P. W. Voorhees. Faceting of a growing crystal surface by surface diffusion. Phys. Rev. E, 67:021606, 2003.

[23] L. F. Shampine, P. H. Muir, and H. Xu. A user-friendly Fortran BVPsolver. Journal of Numerical Analysis, Industrial and Applied Mathematics, 1(2):201-217, 2006.

[24] V. A. Shchukin and D. Bimberg. Spontaneous ordering of nanostructures on crystal surfaces. Rev. Modern Phys., 71(4):1125-1171, July 1999.

[25] M. J. Ward. Eliminating indeterminacy in singularly perturbed boundary value problems with transition invariant potentials. Stud. Appl. Math., 87:95-134, 1992.

[26] S. Watson, F. Otto, B. Rubinstein, and S. Davis. Coarsening dynamics of the convective Cahn-Hilliard equation. Phys. D, 178:127-148, 2003.

[27] C. Yeung, T. Rogers, A. Hernandes-Machado, and D. Jasnow. Phase separation dynamics in driven diffusive systems. J. Statist. Phys., 66:1071-1088, 1992.

[28] M. A. Zaks, A. Podolny, A. A. Nepomnyashchy, and A. A. Golovin. Periodic stationary patterns governed by a convective Cahn-Hilliard equation. SIAM Journal on Applied Mathematics, 66(2):700-720, 2006. 